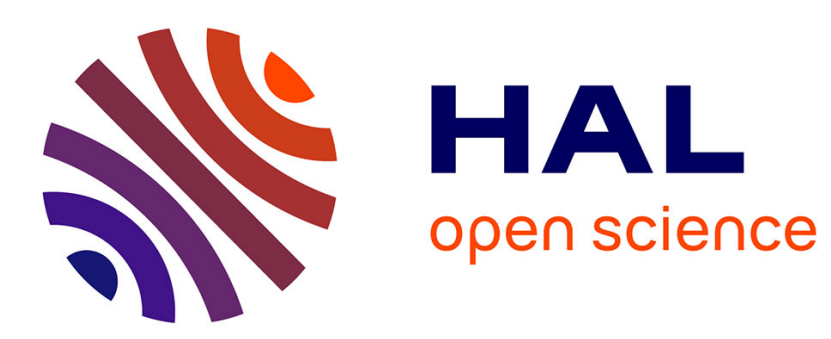

\title{
A High Order Method for the Approximation of Integrals Over Implicitly Defined Hypersurfaces
}

\author{
Lukas Drescher, Holger Heumann, Kersten Schmidt
}

\section{To cite this version:}

Lukas Drescher, Holger Heumann, Kersten Schmidt. A High Order Method for the Approximation of Integrals Over Implicitly Defined Hypersurfaces. SIAM Journal on Numerical Analysis, 2017, 55 (6), pp.2592 - 2615. 10.1137/16M1102227 . hal-01637946

\section{HAL Id: hal-01637946 https://hal.science/hal-01637946}

Submitted on 18 Nov 2017

HAL is a multi-disciplinary open access archive for the deposit and dissemination of scientific research documents, whether they are published or not. The documents may come from teaching and research institutions in France or abroad, or from public or private research centers.
L'archive ouverte pluridisciplinaire HAL, est destinée au dépôt et à la diffusion de documents scientifiques de niveau recherche, publiés ou non, émanant des établissements d'enseignement et de recherche français ou étrangers, des laboratoires publics ou privés. 


\title{
A HIGH ORDER METHOD FOR THE APPROXIMATION OF INTEGRALS OVER IMPLICITLY DEFINED HYPERSURFACES
}

\author{
LUKAS DRESCHER*, HOLGER HEUMANN ${ }^{\dagger}$, AND KERSTEN SCHMIDT ${ }^{\ddagger}$
}

\begin{abstract}
We introduce a novel method to compute approximations of integrals over implicitly defined hypersurfaces. The new method is based on a weak formulation in $L^{2}(0,1)$, that uses the coarea formula to circumvent an explicit integration over the hypersurfaces. As such it is possible to use standard quadrature rules in the spirit of $\mathrm{hp} / \mathrm{spectral}$ finite element methods, and the expensive computation of explicit hypersurface parametrizations is avoided. We derive error estimates showing that high order convergence can be achieved provided the integrand and the hypersurface defining function are sufficiently smooth. The theoretical results are supplemented by numerical experiments including an application for plasma modeling in nuclear fusion.
\end{abstract}

1. Introduction. This article is concerned with the approximation of the integrals

$$
g_{f, \bar{\psi}}(y):=\int_{\{\mathbf{x} \in \Omega, \bar{\psi}(\mathbf{x})=y\}} f(\mathbf{x}) \mathrm{d} s(\mathbf{x}), \quad y \in(0,1),
$$

where $\bar{\psi}: \Omega \subset \mathbb{R}^{n} \rightarrow[0,1], n=2,3$ is a Lipschitz continuous, scalar function, and $f: \Omega \rightarrow \mathbb{R} \cup\{-\infty, \infty\}$ is another integrable scalar function. The values $g_{f, \bar{\psi}}(y)$ are well defined for all $y$ for which the level sets $\left\{\mathbf{x} \in \mathbb{R}^{n}, \bar{\psi}(\mathbf{x})=y\right\}$ are $(n-1)$-dimensional hypersurfaces and $f$ is bounded on these sets. The functions $g_{f, \bar{\psi}}$ represent the average of $f$ over each hypersurface of $\bar{\psi}$ multiplied by the hypersurface area $g_{1, \bar{\psi}}$.

If the hypersurfaces of $\bar{\psi}$ have an explicit parametrization, standard quadrature formulas provide adequate means to approximate (1). It is even possible to devise higher order methods that avoid the computation of the derivatives of the parametrization [3].

In here we want to focus on the case where the hypersurface is a priori not given by an explicit parametrization, but implicitly as level set of a scalar function. The standard approach is the following: The scalar function $\bar{\psi}$ is approximated by a piecewise polynomial finite element function $\bar{\psi}_{h}$, and the values $g_{f, \bar{\psi}_{h}}(y)$ can be assembled from contributions in each element. This approach is a variation of a family of popular algorithms in computer graphics [23] that are used to visualize hypersurfaces. In the case of linear finite elements this is the method of marching triangles or tetrahedrons [24,30], while the case of bilinear finite elements is the older method of marching squares or cubes [19]. To the best of our knowledge, there does not exist rigorous convergence analysis. But clearly the projection of $\bar{\psi}$ onto low order spaces restricts the convergence order of the method. Very high accuracy can be only achieved through meshes with a huge number of elements.

On the other hand it is a well known fact in spectral and higher order methods, that one can find way more economical finite dimensional approximations of $\bar{\psi}$, if one exploits smoothness. Nevertheless, we would like to stress that the direct elementwise assembling of $g_{f, \bar{\psi}_{h}}$ with $\bar{\psi}_{h}$ piecewise polynomials of degree $p$ will be more difficult and expensive: in the case of linear finite elements the intersections of hypersurfaces with the mesh elements are

*CASTOR, Inria Sophia Antipolis - Méditerranée, 2004, route des Lucioles-BP 93, 06902 Sophia Antipolis Cedex, France, and Institut für Mathematik, Technische Universität Berlin, Straße des 17. Juni 136, 10623 Berlin, Germany, lukas.drescher@gmail.com

${ }^{\dagger}$ CASTOR, Inria Sophia Antipolis - Méditerranée, 2004, route des Lucioles-BP 93, 06902 Sophia Antipolis Cedex, France, Holger.Heumann@inria.fr

${ }^{\ddagger}$ Institut für Mathematik, Technische Universität Berlin, Straße des 17. Juni 136, 10623 Berlin, Germany, and Institut für Mathematik, Brandenburgisch Technische Universität Cottbus-Senftenberg, Platz der Deutschen Einheit 1, 03046 Cottbus, Germany, kersten.schmidt@math.tu-berlin.de 
planar, while in the case of higher order finite elements the intersections are curved. Hence, it is necessary to determine numerically a local hypersurface parametrization if the accuracy of $\bar{\psi}_{h}$ shall be inherited to the approximation of $g_{f, \bar{\psi}_{h}}$. Finding such local parametrizations requires extra data structures for geometric refinement and algorithms for repeatedly solving non-linear problems [6, Chapter 12]. The difficulty becomes especially magnified in 3 dimensions. Alternatively one could, as in [25], introduce locally approximated parametrizations based on higher order reconstructions of discretized hypersurfaces. For higher order polynomial approximations of the level sets of $\psi$ both these approaches are fairly expensive and algorithmically involved. Such efforts might be reasonable if one is interested in values of (1) for one specific value $y$ as it is the case for hypersurface integrals that appear in level set methods and fictitious domain methods or extended finite element methods. A non-exhaustive list of references about this topic is $[7,22,10]$ and the many references therein. We also mention the more recent [26], [17] and [14] that address the issue of integrals over implicitly defined hypersurfaces as important ingredient of fictitious domain methods, unfitted finite element methods and numerical methods of partial differential equations on surfaces.

In this article we are interested in computing efficiently approximations of $g_{f, \bar{\psi}}$ in (1) on the whole interval $y \in(0,1)$. We propose a novel approach fitting seamlessly into the spirit of higher order finite element methods. We will see that the hypersurface integrals (1) are unique solutions of the variational formulation: Seek $g_{f, \bar{\psi}} \in L^{2}(0,1)$ such that

$$
\int_{0}^{1} g_{f, \bar{\psi}}(y) \lambda(y) \mathrm{d} y=\int_{\Omega} f(\mathbf{x})|\nabla \bar{\psi}(\mathbf{x})| \lambda(\bar{\psi}(\mathbf{x})) \mathrm{d} \mathbf{x} \quad \forall \lambda \in L^{2}(0,1) .
$$

The main motivation for this work is the magnetohydrodynamic equilibrium equation in axisymmetric models of nuclear fusion reactors, an elliptic semi-linear equation for the so-called poloidal flux $\psi$. Very important functionals of the solution of such equilibrium problems are the hypersurface integrals (1) that are the focus of this article. They are essential to incorporate resistive diffusion effects into plasma evolution modeling. Also many plasma characteristics (e.g. the so-called safety factor or the current density profile), important to quantify stability or for monitoring during the experiment, are defined as integrals over hypersurfaces [11].

The outline is the following. In the next section, we will recall the coarea formula and show (Corollary 2) that the hypersurface integrals satisfy the weak formulation (2). With this, it is straight forward to introduce the $L^{2}$ projection methods to compute approximations of $g_{f, \bar{\psi}}(y)$ in some finite dimensional space, e.g., a polynomial space. We provide also convergence estimates in $L^{2}, H^{1}$ and $L^{\infty}$. The next two sections cover two different extensions of these results addressing important aspects for implementation and applications. Section 3 covers the case where only approximations of the level set function $\psi$ are available and in section 4 we focus on the analysis of the relevant case where quadrature is involved. We want to stress that the analysis of the consistency error due to quadrature is here a considerable evolved extension of the analysis of similar consistency errors in the finite element literature, since we have to deal with weak formulations with non-polynomial test functions. Extensive numerical experiments for a model problem and the plasma equilibrium problem in Section 5 highlight the practicability of the new method.

2. Hypersurface integrals and the coarea formula. We recall that $\bar{\psi}: \Omega \subset \mathbb{R}^{n} \rightarrow$ $(0,1), n=2,3$ is a Lipschitz continuous, scalar function, not necessarily monotone and that the hypersurface integrals are the integrals

$$
g_{f, \bar{\psi}}(y):=\int_{\{\mathbf{x} \in \Omega, \bar{\psi}(\mathbf{x})=y\}} f(\mathbf{x}) \mathrm{d} s(\mathbf{x}),
$$


with $f: \Omega \mapsto \mathbb{R} \cup\{-\infty, \infty\}$ another integrable scalar function. We have the following result, the coarea formula, highlighting the relationship between integrated hypersurface integrals $g_{f, \bar{\psi}}(y)$ and volume integrals.

THEOREM 1 (Coarea formula). Let $f: \Omega \rightarrow \mathbb{R} \cup\{-\infty, \infty\}$ be integrable and $\bar{\psi}: \Omega \rightarrow$ $[0,1]$ Lipschitz, then $g_{f, \bar{\psi}} \in L^{1}(0,1)$ and

$$
\int_{0}^{1} g_{f, \bar{\psi}}(y) \mathrm{d} y=\int_{\Omega} f(\mathbf{x})|\nabla \bar{\psi}(\mathbf{x})| \mathrm{d} \mathbf{x} .
$$

Proof. See [8, Theorem 3.2.12].

An immediate corollary of the coarea formula is the following identity.

COROLLARY 2. Let the assumptions of Theorem 1 be fulfilled. If moreover (i) $f|\nabla \bar{\psi}|^{\frac{1}{2}} \in$ $L^{2}(\Omega)$ and $g_{1, \bar{\psi}} \in L^{\infty}(0,1)$ or (ii) $f|\nabla \bar{\psi}| \in L^{2}(\Omega)$ and $g_{|\nabla \bar{\psi}|^{-1}, \bar{\psi}} \in L^{\infty}(0,1)$ then $g_{f, \bar{\psi}} \in$ $L^{2}(0,1)$ and

$$
\int_{0}^{1} g_{f, \bar{\psi}}(y) \lambda(y) \mathrm{d} y=\int_{\Omega} f(\mathbf{x}) \lambda(\bar{\psi}(\mathbf{x}))|\nabla \bar{\psi}(\mathbf{x})| \mathrm{d} \mathbf{x} \quad \forall \lambda \in L^{2}(0,1) .
$$

Proof. We restrict the proof to the case (i), the case (ii) follows similarly. The assertion follows from the identity $g_{f, \bar{\psi}}(y) \lambda(y)=g_{f \cdot \lambda(\bar{\psi}), \bar{\psi}}(y)$ and Theorem 1 , and so

$$
\int_{0}^{1} g_{f, \bar{\psi}}(y) \lambda(y) \mathrm{d} y=\int_{0}^{1} g_{f \cdot \lambda(\bar{\psi}), \bar{\psi}}(y) \mathrm{d} y=\int_{\Omega} f(\mathbf{x}) \lambda(\bar{\psi}(\mathbf{x}))|\nabla \bar{\psi}(\mathbf{x})| \mathrm{d} \mathbf{x} .
$$

Then, the Cauchy-Schwarz inequality implies

$$
\int_{\Omega} f(\mathbf{x}) \lambda(\bar{\psi}(\mathbf{x}))|\nabla \bar{\psi}(\mathbf{x})| \mathrm{d} \mathbf{x} \leq\left\|f|\nabla \bar{\psi}|^{\frac{1}{2}}\right\|_{L^{2}(\Omega)}\left\|\lambda \circ \bar{\psi}|\nabla \bar{\psi}|^{\frac{1}{2}}\right\|_{L^{2}(\Omega)},
$$

and so the right-hand side of (3) is bounded in $L^{2}(0,1)$ due to

$$
\begin{aligned}
\left\|\lambda \circ \bar{\psi}|\nabla \bar{\psi}|^{\frac{1}{2}}\right\|_{L^{2}(\Omega)}^{2} & =\left|\int_{\Omega} \lambda^{2}(\bar{\psi}(\mathbf{x}))\right| \nabla \bar{\psi}(\mathbf{x})|d \mathbf{x}| \\
& =\left|\int_{0}^{1} g_{1, \bar{\psi}}(y) \lambda^{2}(y) d y\right| \leq\|\lambda\|_{L^{2}(0,1)}^{2}\left\|g_{1, \bar{\psi}}\right\|_{L^{\infty}(0,1)} .
\end{aligned}
$$

Hence, the weak formulation (3) for $g_{f, \bar{\psi}} \in L^{2}(0,1)$ is well-posed and the proof is complete. $\square$

REMARK 3. The assumption $g_{1, \bar{\psi}} \in L^{\infty}(0,1)$ in the first case (i) in Corollary 2 corresponds to assuming bounded area of the hypersurface as is clear from the definition in (1). But also the assumption $g_{|\nabla \bar{\psi}|^{-1}, \bar{\psi}} \in L^{\infty}(0,1)$ in second case (ii) has an intuitive meaning. By the coarea formula we have

$$
\int_{0}^{y} g_{f, \bar{\psi}}\left(y^{\prime}\right) \mathrm{d} y^{\prime}=\int_{\{\mathbf{x} \in \Omega, \bar{\psi}(\mathbf{x}) \leq y\}} f(\mathbf{x})|\nabla \bar{\psi}(\mathbf{x})| \mathrm{d} \mathbf{x},
$$

and hence, $g_{|\nabla \bar{\psi}|^{-1}, \bar{\psi}}$ stands for the differential change of the volume that is enclosed by the level sets $\left\{\mathbf{x} \in \mathbb{R}^{n}, \bar{\psi}(\mathbf{x})=0\right\}$ and $\left\{\mathbf{x} \in \mathbb{R}^{n}, \bar{\psi}(\mathbf{x})=y\right\}$. 
We are going to use the weak formulation (3) to compute $L^{2}$ projections of the hypersurface integrals to discrete spaces, rather than introducing directly discretizations of the strong formulation in (1).

Let us introduce $\mathcal{P}_{P}(0,1)$ the space of all polynomials of degree less or equal to $P$ on $[0,1]$ and look for an approximation $g_{f, \bar{\psi}}^{P} \in \mathcal{P}_{P}(0,1)$ of $g_{f, \bar{\psi}}$ such that

$$
\int_{0}^{1} g_{f, \bar{\psi}}^{P}(y) \lambda(y) \mathrm{d} y:=\int_{0}^{1} g_{f, \bar{\psi}}(y) \lambda(y) \mathrm{d} y \quad \forall \lambda \in \mathcal{P}_{P}(0,1) .
$$

The benefit of this idea is that the coarea formula (Corollary 2) can be used to rewrite the integration over $[0,1]$ in the right hand side of (5) in an integration over $\Omega$. We will work with the equivalent formulation: Find $g_{f, \bar{\psi}}^{P} \in \mathcal{P}_{P}(0,1)$ such that

$$
\int_{0}^{1} g_{f, \bar{\psi}}^{P}(y) \lambda(y) \mathrm{d} y=\int_{\Omega} f(\mathbf{x}) \lambda(\bar{\psi}(\mathbf{x}))|\nabla \bar{\psi}| \mathrm{d} \mathbf{x} \quad \forall \lambda \in \mathcal{P}_{P}(0,1) .
$$

The practical implementation of (5) comprises two steps/ingredients. First, a standard $(P+$ $1) \times(P+1)$ mass matrix containing the $L^{2}$ inner products of pairs of basis functions of $\mathcal{P}_{P}(0,1)$ has to be assembled. This mass matrix becomes diagonal when Legendre polynomials - shifted to $(0,1)$ - are used as basis functions. Secondly, $P+1$ entries of the right hand side vector have to be assembled in an element by element fashion over a triangulation of $\Omega$. However, there is no need to determine explicitly the hypersurfaces of $\psi$.

There are standard well known approximation results for the solution of (5) in $L^{2}, H^{1}$ and $L^{\infty}$ that we restate for completeness.

THEOREM 4 ( $L^{2}$-error). Let the assumptions of Corollary 2 be fulfilled, and assume that $g_{f, \bar{\psi}} \in H^{m}(0,1)$. Then the solution $g_{f, \bar{\psi}}^{P}$ of (5) is well-defined in $L^{2}(0,1)$ and there exists a constant $C=C(m)>0$ that is independent of $P$ such that for all $k \leq m$

$$
\left\|g_{f, \bar{\psi}}^{P}-g_{f, \bar{\psi}}\right\|_{L^{2}(0,1)}=\inf _{v \in \mathcal{P}_{P}(0,1)}\left\|v-g_{f, \bar{\psi}}\right\|_{L^{2}(0,1)} \leq C P^{-k}\left\|g_{f, \bar{\psi}}\right\|_{H^{k}(0,1)} .
$$

Proof. The proof follows directly from the Pythagorean theorem and approximation estimates [28, Theorem 3.17].

COROLlaRY 5 ( $L^{\infty}$ - and $H^{1}$-error). Let the assumptions of Theorem 4 be fulfilled. Then, there exists a constant $C=C(m)>0$ that is independent of $P$ such that for all $k \leq m$

$$
\begin{aligned}
\left|g_{f, \bar{\psi}}^{P}-g_{f, \bar{\psi}}\right|_{H^{1}(0,1)} & \leq C P^{-k+2}\left\|g_{f, \bar{\psi}}\right\|_{H^{k}(0,1)}, \\
\left\|g_{f, \bar{\psi}}^{P}-g_{f, \bar{\psi}}\right\|_{L^{\infty}(0,1)} & \leq C P^{-k+1}\left\|g_{f, \bar{\psi}}\right\|_{H^{k}(0,1)} .
\end{aligned}
$$

Proof. We denote $m$-dependent constants that are independent of $P$ by $C$ throughout this proof. According to [28, Theorem 3.17], [5, Section 16] and the continuous embedding $H^{1}(0,1) \hookrightarrow L^{\infty}(0,1)$ due to the Sobolev embedding theorem [28, Theorem A.21] we have a projection operator $I_{P}: H^{1}(0,1) \rightarrow \mathcal{P}_{P}(0,1)$ such that for all $v \in H^{k}(0,1)$

$$
\begin{aligned}
\left\|v-I_{P} v\right\|_{L^{2}(0,1)} & \leq C P^{-k}\|v\|_{H^{k}(0,1)}, \\
\left\|v-I_{P} v\right\|_{L^{\infty}(0,1)}+\left|v-I_{P} v\right|_{H^{1}(0,1)} & \leq C P^{-k+1}\|v\|_{H^{k}(0,1)} .
\end{aligned}
$$


Combining these results with triangle inequalities, an inverse inequality for polynomials [28, Theorem 3.91] we obtain the estimate in the $H^{1}$-seminorm as

$$
\begin{aligned}
\left|g_{f, \bar{\psi}}^{P}-g_{f, \bar{\psi}}\right|_{H^{1}(0,1)} & \leq\left|g_{f, \bar{\psi}}^{P}-I_{P} g_{f, \bar{\psi}}\right|_{H^{1}(0,1)}+\left|I_{P} g_{f, \bar{\psi}}-g_{f, \bar{\psi}}\right|_{H^{1}(0,1)} \\
& \leq C P^{2}\left\|g_{f, \bar{\psi}}^{P}-I_{P} g_{f, \bar{\psi}}\right\|_{L^{2}(0,1)}+C P^{-k+1}\left\|g_{f, \bar{\psi}}\right\|_{H^{k}(0,1)} \\
& \leq C P^{2}\left\|g_{f, \bar{\psi}}^{P}-g_{f, \bar{\psi}}\right\|_{L^{2}(0,1)}+C P^{-k+2}\left\|g_{f, \bar{\psi}}\right\|_{H^{k}(0,1)},
\end{aligned}
$$

and using $L^{2}$-estimate of Theorem 4. Similarly the estimate in the $L^{\infty}$-norm follows using an inverse inequality (Theorem 17 in Appendix B) for the $L^{\infty}$-norm.

REMARK 6 (Smoothness of hypersurface integrals). To obtain in general hypersurface integrals $g_{f, \bar{\psi}}$ that are continuous in $(0,1)$ and so $H^{k}(0,1)$-regular with $k \geq \frac{1}{2}$ that can be approximated in $\mathcal{P}_{P}(0,1)$ with a convergence rate larger than $\frac{1}{2}$ we have to assume that $\bar{\psi}$ takes almost all values in $[0,1]$. If $\bar{\psi}$ does not take values in a subinterval $I \subset(0,1)$ then the hypersurface integrals are zero for all $y \in I$ and might be discontinuous on $\partial I$. In this case the convergence rate would be below $\frac{1}{2}$.

Another source of limited smoothness of the hypersurface integrals are degenerated hypersurfaces, e.g., the cases where level sets $\left\{\mathbf{x} \in \mathbb{R}^{n}, \bar{\psi}(\mathbf{x})=y_{d}\right\}$ are not (n-1)-dimensional hypersurfaces. For such a value $y_{d}$ the hypersurface integrals are not defined, however, its limits from both sides of $y_{d}$ when $y \rightarrow y_{d}$ may be defined and do not coincide in general. We summarize a partial result due to G. Vigfússon, characterizing the smoothness of hypersurface integrals for level set functions with elliptic points in the Appendix A.

REMARK 7. If one is only interested in the approximation in specific values of $g_{f, \bar{\psi}}(y)$, e.g. at $y=y_{c}, y_{c} \in(0,1)$, as it would be the case for level set methods, fictitious domain method or extended finite element methods, it might be worth to introduce a smooth cutoff function $w_{\varepsilon}: \mathbb{R} \rightarrow \mathbb{R}$ with $w_{\varepsilon}(0)=1$ and compact support $(-\varepsilon, \varepsilon)$. Clearly

$$
g_{f \cdot w_{\varepsilon}}\left(\bar{\psi}-y_{c}\right), \bar{\psi}\left(y_{c}\right)=g_{f, \bar{\psi}}\left(y_{c}\right)
$$

and

$$
\int_{0}^{1} g_{f \cdot w_{\varepsilon}\left(\bar{\psi}-y_{c}\right), \bar{\psi}}(y) \lambda(y) \mathrm{d} y=\int_{\Omega} f(\mathbf{x}) w_{\varepsilon}\left(\bar{\psi}(\mathbf{x})-y_{c}\right) \lambda(\bar{\psi}(\mathbf{x}))|\nabla \bar{\psi}(\mathbf{x})| \mathrm{d} \mathbf{x} .
$$

The integrand in the right hand side of the last equation vanishes in large parts of the domain $\Omega$ which reduces the amount of work required to assemble the right hand side of the weak formulation (10). Alternatively, depending on the application and the concrete setting, it is also possible to pursue a more localized approach, where the global integral $g_{f, \bar{\psi}}(y)$ is split into a sum of integrals

$$
g_{f, \bar{\psi}}\left(y_{c}\right)=\sum_{K \in \mathcal{T}_{h}} g_{f, \bar{\psi}}^{K}\left(y_{c}\right)=\sum_{K \in \mathcal{T}_{h}} \int_{\left\{\mathbf{x} \in \Omega \cap K, \bar{\psi}(\mathbf{x})=y_{c}\right\}} f(\mathbf{x}) d s(\mathbf{x}),
$$

where the summation runs over the elements $K$ of a triangulation $\mathcal{T}_{h}$ of $\Omega$. Exemplary for this are the fictitious domain methods, where one needs to evaluate integrals $g_{f, \bar{\psi}}$ for a large number of different $f$ with local support. Applying our method to the approximation of $g_{f, \bar{\psi}}^{K}\left(y_{c}\right)$ replaces the involved integration over non-polynomial level sets with quadrature on elements $K$ that is in general readily available. 
3. Approximated Level Set Functions. In many applications only an approximation of the level set function will be available. Moreover a rescaling of the level set function will be necessary to ensure that it maps onto the unit interval $[0,1]$. It is therefore useful to quantify precisely the consistency error of the $L^{2}$ projected hypersurface integrals due to the approximation error of the level set function.

In the following we will consider a continuous, bounded scalar function $\psi: \Omega \subset \subset$ $\mathbb{R}^{n} \rightarrow \mathbb{R}$ and introduce the normalization

$$
\bar{\psi}(\mathbf{x})=\frac{\psi(\mathbf{x})-\psi^{0}(\psi)}{\psi^{1}(\psi)-\psi^{0}(\psi)} \in(0,1),
$$

with $\psi^{1}(\psi)=\inf _{\mathbf{x} \in \Omega} \psi(\mathbf{x}), \psi^{0}(\psi):=\sup _{\mathbf{x} \in \Omega} \psi(\mathbf{x})$.

Let $V_{h, p} \subset C^{0}(\Omega)$ be a finite element space defined over a triangulation $\mathcal{T}_{h}$ of $\Omega$, where $h>0$ corresponds to the largest diameter of an element of the triangulation and $p$ to the polynomial degree on the reference element. In the following we think of $\psi_{h, p} \in V_{h, p}$ as approximation of $\psi$ with $\psi_{h, p}: \Omega \rightarrow \mathbb{R}$ and we assume standard approximation results

$$
\left\|\psi-\psi_{h, p}\right\|_{L^{2}(\Omega)} \leq C h^{p+1} \text { and }\left\|\psi-\psi_{h, p}\right\|_{H^{1}(\Omega)} \leq C h^{p} .
$$

So $\psi_{h, p}$ can be for example the $L^{2}$ or another kind of Galerkin projection of $\psi$. We refer to Sec. 5.2, where $\psi_{h, p}$ is the Galerkin projection of the solution $\psi$ of a partial differential equation. In the application of the axisymmetric magnetohydrodynamic equilibrium problem, $\psi$ is the so-called poloidal flux that is solution of an elliptic quasi-linear partial differential equation, the Grad-Shafranov equation.

Let us introduce the weak formulation for the hypersurface integrals of the discrete level set function $\psi_{h, p}$ : Find $g_{f_{h, p}, \bar{\psi}_{h, p}}^{P} \in \mathcal{P}_{P}(0,1)$ such that

$$
\int_{0}^{1} g_{f_{h, p}, \bar{\psi}_{h, p}}(y) \lambda(y) \mathrm{d} y=\int_{\Omega} f_{h, p}(\mathbf{x}) \lambda\left(\bar{\psi}_{h, p}(\mathbf{x})\right)\left|\nabla \bar{\psi}_{h, p}(\mathbf{x})\right| \mathrm{d} \mathbf{x} \quad \forall \lambda \in \mathcal{P}_{P}(0,1) .
$$

We introduced here also $f_{h, p}(\mathbf{x})$ to cover cases where $f$ depends on $\bar{\psi}$ and, hence, $f_{h, p}$ on its approximation. For example $f_{h, p}(\mathbf{x})=\left|\nabla \bar{\psi}_{h, p}(\mathbf{x})\right|^{-1}$ for $f(\mathbf{x})=|\nabla \bar{\psi}(\mathbf{x})|^{-1}$.

The practical implementation of (10) is based on the same two ingredients as the implementation of (5). As $\lambda\left(\bar{\psi}_{h, p}\right)$ is piecewise smooth and polynomial with respect to the mesh underlying the definition of $V_{h, p}$, we can assume here that the right hand side can be computed exactly and focus on the consistency error due to $\psi_{h, p}$.

We have the following well-posedness and convergence results, the latter balancing the best approximation error $\inf _{\lambda \in \mathcal{P}_{P}(0,1)}\left\|\lambda-g_{f, \bar{\psi}}\right\|_{L^{2}(0,1)}$ with the discretization error of $\bar{\psi}_{h, p}$ as specified in the following theorem.

THEOREM 8 ( $L^{2}$-error with discrete level set function). Let the assumptions of Corollary 2, case (ii), be fulfilled and let $f_{h, p}: \Omega \rightarrow \mathbb{R} \cup\{-\infty, \infty\}$ and $f_{h, p}\left|\nabla \bar{\psi}_{h, p}\right| \in L^{2}(\Omega)$. Then the solution $g_{f_{h, p}, \bar{\psi}_{h, p}}^{P}$ of (10) is well-defined in $L^{2}(0,1)$. If moreover $g_{f, \bar{\psi}} \in H^{m}(0,1)$ then there exists a constant $C>0$ such that for all $k \leq m$

$$
\begin{aligned}
\left\|g_{f_{h, p}, \bar{\psi}_{h, p}}^{P}-g_{f, \bar{\psi}}\right\|_{L^{2}(0,1)} \leq & C\left(P^{-k}\left\|g_{f, \psi}\right\|_{H^{k}(0,1)}+\left\|f|\nabla \psi|-f_{h, p}\left|\nabla \psi_{h, p}\right|\right\|_{L^{2}(\Omega)}\right. \\
& \left.+P^{3}\left\|\psi-\psi_{h, p}\right\|_{L^{\infty}(\Omega)}\|f|\nabla \psi|\|_{L^{2}(\Omega)}\left\|g_{|\nabla \bar{\psi}|^{-1}, \bar{\psi}}\right\|_{L^{\infty}(0,1)}^{\frac{1}{2}}\right) .
\end{aligned}
$$


Proof of Theorem 8. The well-posedness of (10) is a simple consequence of Corollary 2 when replacing $f$ and $\bar{\psi}$ by $f_{h, p}$ and $\bar{\psi}_{h, p}$. We introduce the following notation for the exact integrals

$$
I(v)=\int_{\Omega} v(\mathbf{x}) d \mathbf{x} .
$$

To prove the estimate on the discretization error we use the Strang lemma that implies

$$
\begin{aligned}
\left\|g_{f_{h, p}, \bar{\psi}_{h, p}}^{P}-g_{f, \bar{\psi}}\right\|_{L^{2}(0,1)} \leq & 2 \inf _{\lambda \in \mathcal{P}_{P}(0,1)}\left\|\lambda-g_{f, \bar{\psi}}\right\|_{L^{2}(0,1)} \\
& +\sup _{\lambda \in \mathcal{P}_{P}(0,1)} \frac{\left|I(f|\nabla \bar{\psi}| \lambda \circ \bar{\psi})-I\left(f_{h, p}\left|\nabla \bar{\psi}_{h, p}\right| \lambda \circ \bar{\psi}_{h, p}\right)\right|}{\|\lambda\|_{L^{2}(0,1)}},
\end{aligned}
$$

where the first term is bounded using Theorem 4 . Now, using the triangle inequality and the mean value theorem we obtain for the numerator in the second term on the right hand side for some function $\eta: \Omega \rightarrow(0,1)$

$$
\begin{aligned}
& \left|I(f|\nabla \bar{\psi}| \lambda \circ \bar{\psi})-I\left(f_{h, p}\left|\nabla \bar{\psi}_{h, p}\right| \lambda \circ \bar{\psi}_{h, p}\right)\right| \\
& \quad \leq\left|I\left(\left(f|\nabla \bar{\psi}|-f_{h, p}\left|\nabla \bar{\psi}_{h, p}\right|\right) \lambda \circ \bar{\psi}\right)\right|+\left|I\left(f_{h, p}\left|\nabla \bar{\psi}_{h, p}\right|\left(\lambda \circ \bar{\psi}-\lambda \circ \bar{\psi}_{h, p}\right)\right)\right| \\
& \quad \leq\left|I\left(\left(f|\nabla \bar{\psi}|-f_{h, p}\left|\nabla \bar{\psi}_{h, p}\right|\right) \lambda \circ \bar{\psi}\right)\right|+\left|I\left(f_{h, p}\left|\nabla \bar{\psi}_{h, p}\right|\left(\bar{\psi}-\bar{\psi}_{h, p}\right) \lambda^{\prime} \circ \eta\right)\right| .
\end{aligned}
$$

By the coarea formula (3) and the Cauchy-Schwarz inequality we find

$$
\|\lambda \circ \bar{\psi}\|_{L^{2}(\Omega)}^{2}=\int_{\Omega} \lambda^{2} \circ \bar{\psi} d \mathbf{x}=\int_{0}^{1} \lambda^{2} g_{|\nabla \bar{\psi}|^{-1}, \bar{\psi}} d y \leq\|\lambda\|_{L^{2}(0,1)}^{2}\left\|g_{|\nabla \bar{\psi}|^{-1}, \bar{\psi}}\right\|_{L^{\infty}(0,1)},
$$

and

$$
\left\|\left(\lambda^{\prime} \circ \eta\right)\left(\bar{\psi}_{h, p}-\bar{\psi}\right)\right\|_{L^{2}(\Omega)} \leq\left\|\lambda^{\prime}\right\|_{L^{\infty}(0,1)}\left\|\bar{\psi}_{h, p}-\bar{\psi}\right\|_{L^{2}(\Omega)} .
$$

Next, as $\lambda^{\prime}$ is a polynomial of degree $P-1$ (cf. Lemma 17) and with inverse estimates [28, Theorem 3.91] we get

$$
\left\|\lambda^{\prime}\right\|_{L^{\infty}(0,1)} \leq P\left\|\lambda^{\prime}\right\|_{L^{2}(0,1)}, \quad\left\|\lambda^{\prime}\right\|_{L^{2}(0,1)} \leq 2 \sqrt{3} P^{2}\|\lambda\|_{L^{2}(0,1)} .
$$

Hence, using the Cauchy-Schwarz inequality, (13) and (14) in the last line of (11) we find

$$
\begin{gathered}
\left\|g_{f_{h, p}, \bar{\psi}_{h, p}}^{P}-g_{f, \bar{\psi}}\right\|_{L^{2}(0,1)} \leq C\left(P^{-k}\left\|g_{f, \psi}\right\|_{H^{k}(0,1)}+\left\|f|\nabla \bar{\psi}|-f_{h, p}\left|\nabla \bar{\psi}_{h, p}\right|\right\|_{L^{2}(\Omega)}\right. \\
\left.+P^{3}\left\|\bar{\psi}-\bar{\psi}_{h, p}\right\|_{L^{2}(\Omega)}\left\|f_{h, p}\left|\nabla \bar{\psi}_{h, p}\right|\right\|_{L^{2}(\Omega)}\right) .
\end{gathered}
$$

Now, applying Lemma 19 with $\psi_{+}=\psi, f_{+}=f, \psi_{-}=\psi_{h, p}$ and $f_{-}=f_{h, p}$ and the fact that we have an upper bound of $1 /\left(\psi^{0}\left(\bar{\psi}_{h, p}\right)-\psi^{1}\left(\bar{\psi}_{h, p}\right)\right)$ and that we can bound $\left\|f_{h, p}\left|\nabla \psi_{h, p}\right|\right\|_{L^{2}(\Omega)}$ by a multiple of $\|f|\nabla \psi|\|_{L^{2}(\Omega)}$ the assertion of the theorem follows.

REMARK 9. Our numerical experiments in Section 5.2 indicate that the high powers of $P$ in the bound of Theorem 8 are by far too pessimistic.

Similarly as in the proof of Corollary 5 we can use the $L^{2}$-estimate in Theorem 8 to obtain estimates in the $H^{1}$-seminorm and the $L^{\infty}$-norm. 
COROLlaRY 10 ( $L^{\infty}$ and $H^{1}$-error with discrete level set function). Let the assumptions of Theorem 8 be fulfilled. Then, there exists a constant $C>0$ independent of $P$ such that for all $k \leq m$,

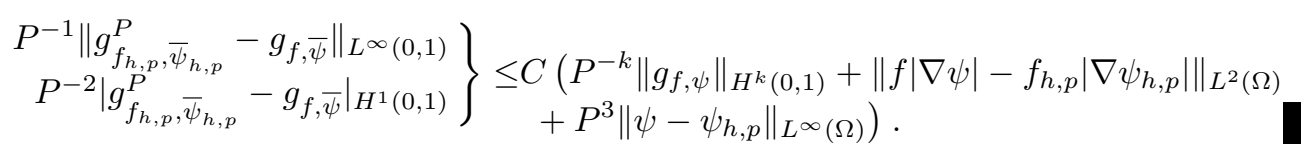

REMARK 11. It is straightforward to replace $\mathcal{P}_{P}(0,1)$ in (10) with some other finite dimensional approximation space, such as spline spaces, for which we can prove a similar convergence results as in Theorem 4. On the other hand it is a priori not obvious, whether, due to the lack of regularity of $\lambda(\bar{\psi})$, the standard quadrature on elements of the mesh $\mathcal{T}_{h}$ underlying the definition of $V_{h, p}$ is sufficient to establish an implementable method.

4. Quadrature. A practical implementation of (5) requires to detail the approximation of the right hand side for the case of non-polynomial level set functions. At first glance, we could use the results from the previous section to introduce a practical method for arbitrary level set functions, in simply approximating the level set functions by functions in some finite element space $V_{h, p}$. But there are two objections: First, the projection introduces an additional step in the implementation and second, the analysis requires that the quadrature is exact for polynomials of degree $p+p P+(p-1)$. The main result of this section, the convergence assertion in Theorem 13, shows that a projection step is not necessary and that, moreover, we can obtain convergence estimates for less expensive quadrature rules.

More precisely, the subsequent analysis addresses the following formulation: Find $\widetilde{g}_{f, \bar{\psi}}^{P}$ $\mathcal{P}_{P}(0,1)$ such that

$$
\int_{0}^{1} \widetilde{g}_{f, \bar{\psi}}^{P}(y) \lambda(y) \mathrm{d} y=Q_{N}(f \lambda \circ \bar{\psi}|\nabla \bar{\psi}|) \quad \forall \lambda \in \mathcal{P}_{P}(0,1),
$$

with

$$
Q_{N}(v)=\sum_{j=1}^{N} \omega_{j} v\left(\mathbf{x}_{j}\right)
$$

where $N$ is the number of quadrature points $\mathbf{x}_{j} \in \bar{\Omega}$ and weights $\omega_{j} \in \mathbb{R}$.

THEOREM 12 (Well-posedness in the case of quadrature). Let the assumptions of Theorem 1 be fulfilled and let $f|\nabla \psi| \in L^{\infty}(\Omega)$. Then, the solution $\widetilde{g}_{f, \bar{\psi}}^{P}$ of (15) is well-defined in $L^{2}(0,1)$ and

$$
\left\|\widetilde{g}_{f, \bar{\psi}}^{P}\right\|_{L^{2}(0,1)} \leq C(P+1)\|f|\nabla \psi|\|_{L^{\infty}(\Omega)},
$$

for some constant $C>0$ independent of $P$ that does not depend on $N$ neither if the quadrature formula (15b) is asymptotically exact for constants for $N \rightarrow \infty$, i.e., $\lim _{N \rightarrow \infty} Q_{N}(1)=$ $|\Omega|$.

Proof. The variational formulation (15) differs from (5) only by its right hand side. It suffices for its well-posedness to show that the right hand side is bounded in $L^{2}(0,1)$. Let us call $\boldsymbol{\omega} \in \mathbb{R}^{N}$ the vector of weights $\omega_{j}, j=1, \ldots, N$ that is bounded in $\ell_{1}$ for any $N \in \mathbb{R}$. 
Hence,

$$
\begin{aligned}
Q_{N}(f \lambda \circ \bar{\psi}|\nabla \bar{\psi}|) & =\sum_{j=1}^{N} \omega_{j} f\left(\mathbf{x}_{j}\right) \lambda\left(\bar{\psi}\left(\mathbf{x}_{j}\right)\right)\left|\nabla \bar{\psi}\left(\mathbf{x}_{j}\right)\right| \leq\|\boldsymbol{\omega}\|_{\ell_{1}}\|f|\nabla \bar{\psi}|\|_{L^{\infty}(\Omega)}\|\lambda\|_{L^{\infty}(0,1)} \\
& \leq(P+1)\|\boldsymbol{\omega}\|_{\ell_{1}}\|f|\nabla \bar{\psi}|\|_{L^{\infty}(\Omega)}\|\lambda\|_{L^{2}(0,1)},
\end{aligned}
$$

using a well-known results on polynomials in the last step (see Lemma 17).

Now, the Lax-Milgram lemma implies

$$
\left\|\widetilde{g}_{f, \bar{\psi}}^{P}\right\|_{L^{2}(0,1)} \leq(P+1)\|\boldsymbol{\omega}\|_{\ell_{1}}\|f|\nabla \psi|\|_{L^{\infty}(\Omega)} .
$$

Since $Q_{N}(1)=\|\boldsymbol{\omega}\|_{\ell_{1}}$ and with the assumption that $\lim _{N \rightarrow \infty} Q_{N}(1)=|\Omega|$ there exists an upper constant of $\|\boldsymbol{\omega}\|_{\ell_{1}}$ for any $N \in \mathbb{N}$ and the estimate (16) holds with a constant $C$ independent of $N$. This completes the proof.

Given well-posedness of (15), we provide an error estimate taking into account an additional consistency error due to quadrature in the evaluation of the integrals of the right hand side. We will address the case of quadrature based on parametrically defined local quadrature rules. Which means, we assume that there exists a triangulation $\mathcal{T}_{h}$ of $\Omega$ with mesh-width $h:=\max _{K \in \mathcal{T}_{h}} \operatorname{diam}(K)$, where each element $K$ is the image of a reference element $\widehat{K}$ under an affine mapping $\Phi_{K}: \widehat{K} \mapsto K$, i.e.,

$$
Q_{N}(v)=\sum_{K \in \mathcal{T}_{h}} \sum_{j=1}^{R_{K}} \omega_{j, K} v\left(\mathbf{x}_{j, K}\right),
$$

with, e.g., $\omega_{j, K}=\widehat{\omega}_{j}|K|$ and $\mathbf{x}_{j, K}=\Phi_{K}\left(\widehat{\mathbf{x}}_{j}\right)$ where $\widehat{\omega}_{j}$ and $\widehat{\mathbf{x}}_{j}$ are weights and quadrature points in $\widehat{K}$.

THEOREM 13 ( $L^{2}$-error in the case of quadrature). Let the assumptions of Corollary 2, case (ii), be fulfilled, $g_{f, \bar{\psi}} \in H^{m}(0,1), m>0, \bar{\psi} \in H^{s+1}(\Omega), s>1-\frac{n}{2}$, where $n$ is the space dimension, and $f|\nabla \bar{\psi}| \in W^{r, \infty}(\Omega), r \geq 1$ and, $Q_{N}$ in (15) is based on a parametrically defined local quadrature rule (17) that is exact for polynomials up to order $q$, then there exists for all $p<s$ with $r \geq q-p P+1>0$, a constant $C>0$ independent of $P$ and $h$ but dependent of $m, s$ such that the solution $\widetilde{g}_{f, \bar{\psi}}^{P}$ of $(15)$ is well-defined in $L^{2}(0,1)$ and for all $k \leq m$,

$$
\begin{aligned}
\left\|\widetilde{g}_{f, \bar{\psi}}^{P}-g_{f, \bar{\psi}}\right\|_{L^{2}(0,1)} \leq C( & P^{-k}\left\|g_{f, \bar{\psi}}\right\|_{H^{k}(0,1)}+P^{3} h^{p+1-\frac{n}{2}}\|f|\nabla \bar{\psi}|\|_{L^{\infty}(\Omega)}\|\bar{\psi}\|_{H^{p+1}(\Omega)} \\
& \left.+h^{q-p P+1}\|f|\nabla \bar{\psi}|\|_{W^{q-p P+1, \infty}(\Omega)}\left\|g_{|\nabla \bar{\psi}|^{-1}, \bar{\psi}}\right\|_{L^{\infty}(0,1)}^{\frac{1}{2}}\right)
\end{aligned}
$$

Even though Theorem 13 is limited to parametrically defined local quadrature with affine mappings $\Phi_{K}$, as shown in the following proof, the extension to $C^{\infty}$-mappings is only technical.

Proof. With the assumption on $f$ and $\bar{\psi}$ and in view of Theorem 12 the approximated hypersurface integral $\widetilde{g}_{f, \bar{\psi}}^{P}$ is well-defined in $L^{2}(0,1)$. We recall the notation for the exact integrals

$$
I(v)=\int_{\Omega} v(\mathbf{x}) d \mathbf{x}
$$


To prove the estimate on the discretization error we use the Strang lemma that yields (18)

$$
\left\|\widetilde{g}_{f, \bar{\psi}}^{P}-g_{f, \bar{\psi}}\right\|_{L^{2}(0,1)} \leq 2 \inf _{\lambda \in \mathcal{P}_{P}(0,1)}\left\|\lambda-g_{f, \bar{\psi}}\right\|_{L^{2}(0,1)}+\sup _{\lambda \in \mathcal{P}_{P}(0,1)} \frac{\left|\left(I-Q_{N}\right)(f|\nabla \bar{\psi}| \lambda \circ \bar{\psi})\right|}{\|\lambda\|_{L^{2}(0,1)}}
$$

The first term on the right hand side of (18) can be bounded using Theorem 4 and the second term is the consistency error due to quadrature. The function $\lambda \circ \bar{\psi}$ is not in general a polynomial on the elements $K$ of the triangulation $\mathcal{T}_{h}$ and we prefer to estimate a similar term where $\bar{\psi}$ is replaced by a discrete $\mathcal{T}_{h}$-piecewise polynomial approximation $\bar{\psi}_{h, p}^{c}$ of uniform polynomial degree $p \leq s$ that we will specify later. Then, on each cell $K$ of $\mathcal{T}_{h}$ the function $\lambda \circ \bar{\psi}_{h, p}^{c}$ is a polynomial of degree $p P$ (in each direction). Using the Cauchy-Schwarz inequality and a Bramble-Hilbert perturbation argument for local parametric quadrature (see Theorem 16) we get

$$
\left|\left(I-Q_{N}\right)\left(f|\nabla \bar{\psi}| \lambda \circ \bar{\psi}_{h, p}^{c}\right)\right| \leq C h^{q-p P+1}\|f|\nabla \bar{\psi}|\|_{W^{q-p P+1, \infty}(\Omega)}\left\|\lambda \circ \bar{\psi}_{h, p}^{c}\right\|_{L^{2}(\Omega)} .
$$

With the mean value theorem, stating that there is some function $\eta: \Omega \rightarrow(0,1)$ such that

$$
\lambda(\bar{\psi}(\mathbf{x}))-\lambda\left(\bar{\psi}_{h, p}^{c}(\mathbf{x})\right)=\lambda^{\prime}(\eta(\mathbf{x}))\left(\bar{\psi}(\mathbf{x})-\bar{\psi}_{h, p}^{c}(\mathbf{x})\right) \quad \forall \mathbf{x} \in \Omega,
$$

and applying the triangle inequality we can bound

$$
\left\|\lambda \circ \bar{\psi}_{h, p}^{c}\right\|_{L^{2}(\Omega)} \leq\|\lambda \circ \bar{\psi}\|_{L^{2}(\Omega)}+\left\|\left(\lambda^{\prime} \circ \eta\right)\left(\bar{\psi}_{h, p}^{c}-\bar{\psi}\right)\right\|_{L^{2}(\Omega)} .
$$

So altogether, recalling (12), (13) and (14) we obtain

$$
\begin{aligned}
& \sup _{\lambda \in L^{2}(0,1)} \frac{\left|\left(I-Q_{N}\right)\left(f|\nabla \bar{\psi}| \lambda \circ \bar{\psi}_{h, p}^{c}\right)\right|}{\|\lambda\|_{L^{2}(0,1)}} \\
& \leq C h^{q-p P+1}\|f|\nabla \bar{\psi}|\|_{W^{q-p P+1, \infty}(\Omega)}\left(\left\|g_{|\nabla \bar{\psi}|^{-1}, \bar{\psi}}\right\|_{L^{\infty}(0,1)}^{\frac{1}{2}}+P^{3}\left\|\bar{\psi}_{h, p}^{c}-\bar{\psi}\right\|_{L^{2}(\Omega)}\right) .
\end{aligned}
$$

As we have studied the consistency error with the piecewise polynomial $\bar{\psi}_{h, p}^{c}$ instead of $\bar{\psi}$ it remains to bound

$$
\begin{aligned}
\left|\left(I-Q_{N}\right)\left(f|\nabla \bar{\psi}|\left(\lambda \circ \bar{\psi}-\lambda \circ \bar{\psi}_{h, p}^{c}\right)\right)\right| & =\left|\left(I-Q_{N}\right)\left(f|\nabla \bar{\psi}| \lambda^{\prime} \circ \eta\left(\bar{\psi}-\bar{\psi}_{h, p}^{c}\right)\right)\right| \\
& \leq C\|f|\nabla \bar{\psi}|\|_{L^{\infty}(\Omega)}\left\|\lambda^{\prime}\right\|_{L^{\infty}(0,1)}\left\|\bar{\psi}-\bar{\psi}_{h, p}^{c}\right\|_{L^{\infty}(\Omega)} \\
& \leq C P^{3}\|f \mid \nabla \bar{\psi}\|_{L^{\infty}(\Omega)}\|\lambda\|_{L^{2}(0,1)}\left\|\bar{\psi}-\bar{\psi}_{h, p}^{c}\right\|_{L^{\infty}(\Omega)},
\end{aligned}
$$

where similarly to the proof of Theorem 12 the constant $C$ does not depend on $N$ as the quadrature is asymptotically exact for $h \rightarrow 0$ and so $N \rightarrow \infty$.

To complete the proof we need to specify $\bar{\psi}_{h, p}^{c}$ as an approximation to $\bar{\psi}$. Now, we denote by $\bar{\psi}_{h, p}$ a suitable projection of $\bar{\psi}$ onto the Lagrangian finite element space of polynomial degree $p \leq s$ over the triangulation $\mathcal{T}_{h}$ [4]. As $\bar{\psi}_{h, p}$ might exceed $[0,1]$ we define $\bar{\psi}_{h, p}^{c}$ such that

$$
\frac{\bar{\psi}_{h, p}^{c}(\mathbf{x})-\psi^{1}(\bar{\psi})}{\psi^{0}(\bar{\psi})-\psi^{1}(\bar{\psi})}=\frac{\bar{\psi}_{h, p}(\mathbf{x})-\psi^{1}\left(\bar{\psi}_{h, p}\right)}{\psi^{0}\left(\bar{\psi}_{h, p}\right)-\psi^{1}\left(\bar{\psi}_{h, p}\right)}
$$

where $\psi^{0}$ and $\psi^{1}$ denote the supremum and infimum in $\Omega$, respectively. Hence, the ranges of $\bar{\psi}$ and $\bar{\psi}_{h, p}^{c}$ coincide and $\bar{\psi}_{h, p}^{c}(\mathbf{x}) \in[0,1]$ for all $\mathbf{x} \in \Omega$. Now, with the equality

$$
\bar{\psi}_{h, p}^{c}-\bar{\psi}=\left(\frac{\bar{\psi}_{h, p}-\psi^{1}\left(\bar{\psi}_{h, p}\right)}{\psi^{0}\left(\bar{\psi}_{h, p}\right)-\psi^{1}\left(\bar{\psi}_{h, p}\right)}-\frac{\bar{\psi}-\psi^{1}(\bar{\psi})}{\psi^{0}(\bar{\psi})-\psi^{1}(\bar{\psi})}\right)\left(\psi^{0}(\bar{\psi})-\psi^{1}(\bar{\psi})\right)
$$


and Lemma 19, we find that

$$
\left\|\bar{\psi}-\bar{\psi}_{h, p}^{c}\right\|_{L^{\infty}(\Omega)} \leq C\left\|\bar{\psi}-\bar{\psi}_{h, p}\right\|_{L^{\infty}(\Omega)} .
$$

By the assumption $\bar{\psi} \in H^{s+1}(\Omega)$ there is a $\bar{\psi}_{h, p}$, e.g. the interpolation of $\bar{\psi}$ onto the Lagrangian finite element space of polynomial degree $p \leq s$ over the triangulation $\mathcal{T}_{h}$, such that (see e.g. [5, Section 16])

$$
\left\|\bar{\psi}-\bar{\psi}_{h, p}\right\|_{L^{\infty}(\Omega)} \leq C h^{p+1-\frac{n}{2}}\|\bar{\psi}\|_{H^{p+1}(\Omega)} .
$$

Combining the previous estimate we conclude with the statement of the theorem.

5. Numerical Experiments and Applications. In this section, we present numerical results for an example problem and a very relevant application from fusion science that allow to study the influence of the discretisation parameters and support the theoretical results in the previous sections.

5.1. Numerical Experiments. We choose $\Omega=[-1,1]^{2}$ and $\bar{\psi}(\mathbf{x})=\left(x_{1}^{2}-1\right)\left(x_{2}^{2}-1\right)$. By symmetry arguments one establishes

$$
g_{f, \bar{\psi}}(y)=4 \int_{-\sqrt{1-\sqrt{y}}}^{\sqrt{1-\sqrt{y}}} \sqrt{1+\frac{y^{2} x_{1}^{2}}{\left(x_{1}^{2}-1\right)^{3}\left(x_{1}^{2}-1+y\right)}} f\left(x_{1}, \frac{\sqrt{y+x_{1}^{2}-1}}{\sqrt{x_{1}^{2}-1}}\right) d x_{1}
$$

so we can use standard numerical quadrature to find arbitrarily accurate approximations of $g_{f, \bar{\psi}}(s)$ that we use as reference solution to validate the new method. We are performing experiments with $f=f_{i}, i=1,2,3$ for $f_{1}=|\nabla \bar{\psi}|, f_{2}=1$, and $f_{3}=|\nabla \bar{\psi}|^{-1}$. The sketches in Figure 1 indicate that the hypersurface integrals may lose regularity in the vicinity of 0 and 1 corresponding to points where $\bar{\psi}$ attains stationary points, i.e. at $y$ with $\bar{\psi}(\mathbf{x})=y$ and $\nabla \psi(\mathbf{x})=0$.

5.1.1. Global quadrature. In our first experiment we approximate $g_{f_{i}, \bar{\psi}}$ by the method (15), where we use very high order tensor product Gauss-Legendre quadrature with $N=$ $(2 P+1)^{2}$ quadrature points to evaluate the integral over $\Omega$ on the right hand side of (15). We would like to stress that with this choice the right hand sides of (15) for $g_{f_{1}, \bar{\psi}}$ and $g_{f_{3}, \bar{\psi}}$ are exactly evaluated and the consistency error due to quadrature, hence, does vanish. The experiments confirm high order convergence that is limited only by the smoothness of $g_{f_{i}, \bar{\psi}}$, as stated in the convergence estimate of Corollary 5 (see Figure 1, bottom left).

To demonstrate the capability of superalgebraic convergence, we study the method with $f=\omega_{s} f_{i}, i=1,2,3$ with the cutoff function

$$
\omega_{s}(\mathbf{x})= \begin{cases}|\cos (\pi(\bar{\psi}(\mathbf{x})-0.5))|^{s} & \bar{\psi}(\mathbf{x}) \in[0,1] \\ 0 & \text { else }\end{cases}
$$

that improves the regularity in the vicinity of $\bar{\psi}=0,1$ while keeping the hypersurface integral at $y=0.5$. More precisely, it holds $g_{\omega_{s} f_{i}, \bar{\psi}}(0.5)=g_{f_{i}, \bar{\psi}}(0.5)$ and for appropriate choices $s \in \mathbb{N}$ we can ensure $g_{\omega_{s} f_{i}, \bar{\psi}} \in H^{k}(0,1)$ even if $g_{f_{i}, \bar{\psi}} \notin H^{k}(0,1)$. We observe a remarkable increase of the convergence of the error for all three functions $f_{i}, i=1,2,3$ (see Figure 1, bottom right). Indeed, the error seems to converge even superalgebraically in the polynomial degree $P$ which agrees exactly with the convergence estimate in Theorem 4 .

The convergence estimate in Theorem 13 suggests that the approximation error blows up when we increase $P$ while keeping the order of the quadrature rule fixed. To study this 

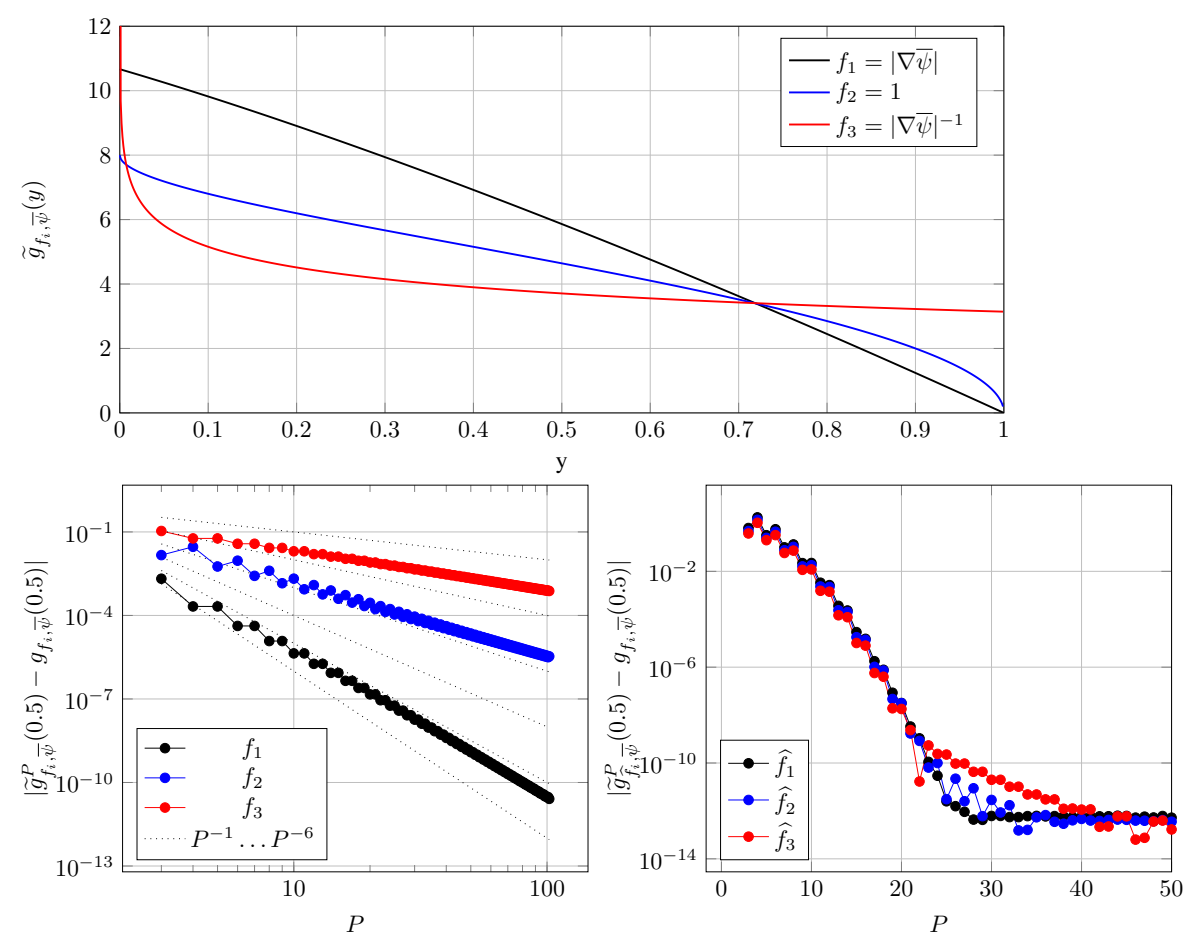

FIG. 1. Convergence in $P$ for quadrature of $P$-adapted order. Top left: Sketch of the hypersurface integrals $\widetilde{g}_{f_{i}, \bar{\psi}}$ for $f_{1}=|\nabla \bar{\psi}|, f_{2}=1$, and $f_{3}=|\nabla \bar{\psi}|^{-1}$. Bottom left: Quadrature with $N=(2 P+1)^{2}$ points. Algebraic convergence is limited by the global regularity of $\widetilde{g}_{f_{i}, \bar{\psi}}$. The visually encountered high (low) regularity of $\widetilde{g}_{f_{1}, \bar{\psi}}$ $\left(\widetilde{g}_{f_{3}, \bar{\psi}}\right)$ is confirmed. Bottom right: To overcome the regularity constraints we redo the numerical experiment with $\widehat{f}_{i}=\omega_{5} f_{i}$ with $\omega_{5}$ as in (25), use quadrature with $N=(2 P+1)^{2}$ points and observe superalgebraic convergence.

effect, we monitor the error as a function of the polynomial degree $P$ for several fixed number of quadrature points $N$ in $[-1,1]^{2}$ (see Figure 2). As expected, the error decreases first for increasing values of $P$, but for $P$ approximately larger than $\sqrt{N}$ the error increases again with a very fast rate.

5.1.2. Local quadrature. To study the influence of parametrically defined local quadrature we introduce a triangulation $\mathcal{T}_{h}$ of rectangular elements $K$ of diameter $h$ and use on each element a Gauss-Legendre quadrature to approximate the right hand side in (5). With this quadrature the hypersurface integral $\widetilde{g}_{f, \bar{\psi}}^{P} \in \mathcal{P}_{P}(0,1)$ solves (15), where $Q_{N}$ is of the form (17). In Figure 3 we keep the polynomial degree $P$ fixed and study the influence of the quadrature order and refinement of the quadrature mesh. We observe high-order convergence in the number of quadrature points up to a saturation that corresponds to the discretization error $\left|g_{\widehat{f}_{i}, \bar{\psi}}^{P}(0.5)-g_{f_{i}, \bar{\psi}}(0.5)\right|$. The results indicate that the refinement of the mesh underlying the quadrature rule leads to a convergence that does not deteriorate when increasing the polynomial order $P$.

5.2. Application: the axisymmetric plasma equilibrium problem. For the application of the axisymmetric magnetohydrodynamic equilibrium problem, $\psi$ is the so-called 

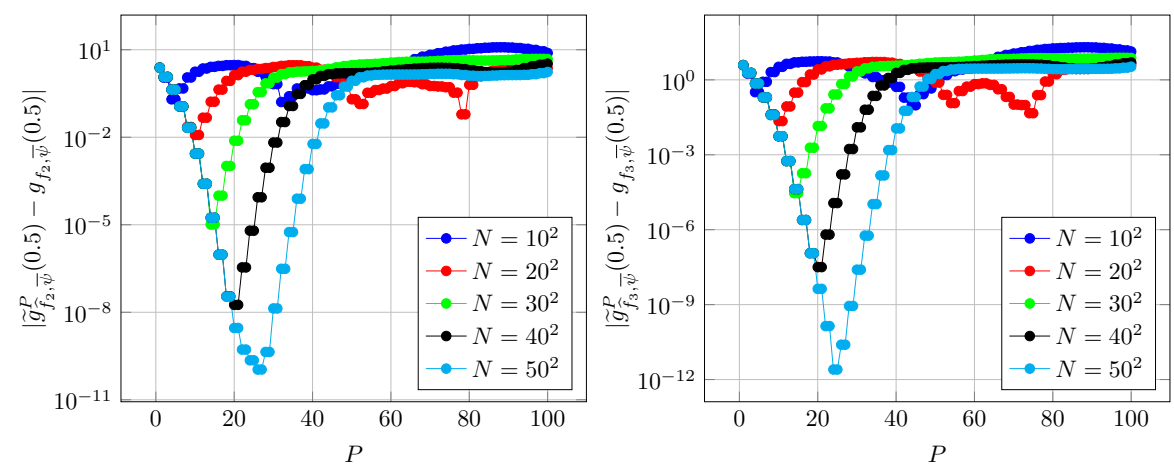

FIG. 2. Convergence in $P$ for quadrature of fixed number of quadrature points $N=10^{2}, N=20^{2}, N=30^{2}$, $N=40^{2}, N=50^{2}$ for the test case $\widehat{f}_{2}=\omega_{5} f_{2}$ (left) and $\widehat{f_{3}}=\omega_{5} f_{3}$ (right). We observe that the error decreases superalgebraic for refinement of $P$ until a certain level and increases afterwards. The optimal values of $P$ are approximately $5,10,15,20,25$.
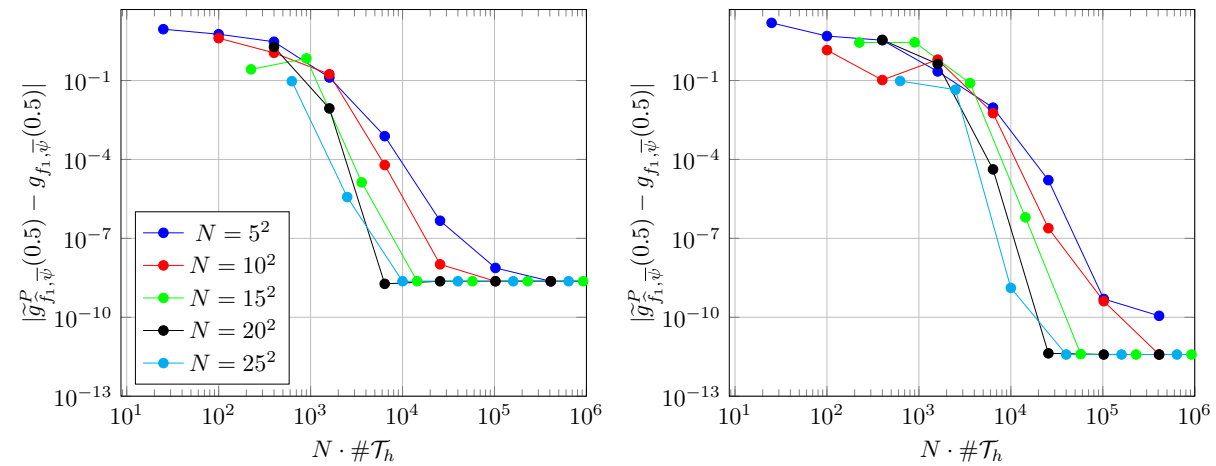

FIG. 3. Convergence when refining the quadrature mesh for a fixed number $N$ of quadrature points per element and fixed polynomial degree $P=20$ (left) and $P=30$ (right) and $\widehat{f}_{1}=\omega_{5} f_{1}$.

poloidal flux and solves the following elliptic quasi-linear partial differential equation:

$$
\begin{aligned}
-\nabla \cdot\left(\frac{1}{\mu_{0} r} \nabla \psi(r, z)\right) & =j(r, \bar{\psi}) \quad \text { in } \Omega ; \\
\psi(r, z) & =\psi_{\mathrm{bd}} \quad \text { at } \partial \Omega ;
\end{aligned}
$$

with $\psi_{\text {bd }} \in \mathbb{R}, \nabla$ the gradient in two dimensions, $\Omega \subset(0, \infty) \times(-\infty, \infty)$ and $\mu_{0}$ the constant magnetic permeability of the vacuum. The right hand side $j(r, \bar{\psi})$ is positive, but in general non-linear in $\bar{\psi}$. Hence, by the maximum principle we have $\psi^{1}=\inf _{(r, z) \in \Omega} \psi(r, z)=\psi_{\text {bd }}$. The equation (26) is the Grad-Shafranov-Schlüter equation [13, 29, 20], that is one of the central equations for modeling of magnetically confined plasma in tokamaks. The physical properties of the plasma, characteristic time and length scales, are highly different for directions parallel and perpendicular to levelsets of $\psi$. Hence, the modeling of plasma equilibrium evolution in tokamaks needs to take into account the very high anisotropy between these two directions. This is achieved in discretizing a hypersurface-integrated version of the magnetohydrodynamic (MHD) equations [12], instead of the full MHD equations in three dimensions. This procedure [15, Chapter 6] is equivalent to rewriting MHD equations in the curvi-linear coordinate system that is induced by the level sets of the poloidal flux function $\psi$ and assuming symmetry in the $\psi$-parallel direction. Some entries of the metric tensor of this non-linear 
change of variables are related to the hypersurface integrals (1) for $n=2, \mathrm{~d} s(\mathbf{x})=r \mathrm{~d} s$ and $\mathrm{d} \mathbf{x}=r \mathrm{~d} r \mathrm{~d} z$. More precisely, these entries, the so-called geometric coefficients $c_{\cdot, \bar{\psi}}(y)$, are given as

$$
c_{f, \bar{\psi}}(y)=g_{f \cdot|\nabla \bar{\psi}|^{-1}, \bar{\psi}}(y) .
$$

For this reason, the modeling of transient plasma equilibrium evolution it is essential to be able to compute such hypersurface integrals with high precision.

We have implemented the Galerkin method for (26) for the linear case $j(r, \bar{\psi}(r, z))=$ $j(r, z)$ in CONCEPTS $[9,27,34]$ (www.concepts.math.ethz.ch), using finite dimensional $h p$ FEM spaces $V_{h, p}$ for the approximations $\psi_{h, p}$ of $\psi$. CONCEPTS provides tensor product basis functions based on integrated Legendre polynomials on the reference square. We make use of CONCEPTS' ability to resolve arbitrary curved boundaries with known parametrization exactly in the mesh by transfinite interpolation techniques. We consider smooth domains and functions $j$ such that the solution $\psi$ of (26) is approximated by Galerkin discretization $\psi_{h, p} \in V_{h, p}$ as

$$
\left\|\psi-\psi_{h, p}\right\|_{L^{2}(\Omega)} \leq C h^{p+1} \quad \text { and } \quad\left\|\psi-\psi_{h, p}\right\|_{H^{1}(\Omega)} \leq C h^{p} .
$$

with constants $C>0$ independent of $h$ and $p$ [28]. Moreover we have the pointwise estimates [5, Section 22]

$$
\left\|\psi-\psi_{h, p}\right\|_{L^{\infty}(\Omega)} \leq C \begin{cases}h^{2}|\log (h)|^{\frac{2}{3}} & p=1 \\ h^{p+1} & p>1 .\end{cases}
$$

Next, fixing the polynomial degree $P$ of the approximation $\widetilde{c}_{f, \bar{\psi}}^{P}(y)=\widetilde{g}_{f \cdot|\nabla \bar{\psi}|^{-1}, \bar{\psi}}^{P}(y)$ of the geometric coefficients, the Theorem 8 and Corollary 10 assert

$$
\left.\begin{array}{l}
\left\|\widetilde{c}_{f_{h, p}, \bar{\psi}_{h, p}}-c_{f, \bar{\psi}}\right\|_{L^{2}(0,1)} \\
\left\|\widetilde{c}_{f_{h, p}, \bar{\psi}_{h, p}}^{P}-c_{f, \bar{\psi}}\right\|_{H^{1}(0,1)}
\end{array}\right\}= \begin{cases}O\left(h^{2}|\log (h)|^{\frac{2}{3}}\right) & p=1, \\
O\left(h^{p+1}\right) & p>1,\end{cases}
$$

for the $\widetilde{c}_{f_{h, p}, \bar{\psi}_{h, p}}^{P}$ with $f_{h, p}=f=1 / r^{2}$ and

$$
\left.\begin{array}{l}
\left\|\widetilde{c}_{f_{h, p}, \bar{\psi}_{h, p}}^{P}-c_{f, \bar{\psi}}\right\|_{L^{2}(0,1)} \\
\left\|\widetilde{c}_{f_{h, p}, \bar{\psi}_{h, p}}^{P}-c_{f, \bar{\psi}}\right\|_{H^{1}(0,1)}
\end{array}\right\}=O\left(h^{p}\right),
$$

with $f=|\nabla \psi|^{2} / r^{2}$ and $f_{h, p}=\left|\nabla \psi_{h, p}\right|^{2} / r^{2}$. Hence, once we invest reasonable amount of effort to achieve high order accuracy for the approximations of $\psi$, we will be rewarded with a likewise high order accuracy for the approximation of the geometric coefficients.

5.2.1. Numerical Experiment: Elliptic Equilibrium. We consider a model problem with the data $\psi^{\mathrm{bd}}=0$ and $j(r, z)=\frac{1}{\mu_{0}}\left(\frac{2}{r}+\frac{2}{9 r}-\frac{2(r-2)}{r^{2}}\right)$ and

$$
\Omega=\left\{\left(\begin{array}{c}
r_{\mathrm{E}}(s, t) \\
z_{\mathrm{E}}(s, t)
\end{array}\right)=\left(\begin{array}{c}
2+\sqrt{2-s} \cos (\pi t) \\
3 \sqrt{2-s} \sin (\pi t)
\end{array}\right) ;(s, t) \in[0,2]^{2}\right\}
$$

an ellipse with center $(2,0)$ and minor and major radius $\sqrt{2}$ and $3 \sqrt{2}$. The solution is given by

$$
\psi_{\mathrm{E}}(r, z)=2-\left((r-2)^{2}+\frac{z^{2}}{9}\right)
$$



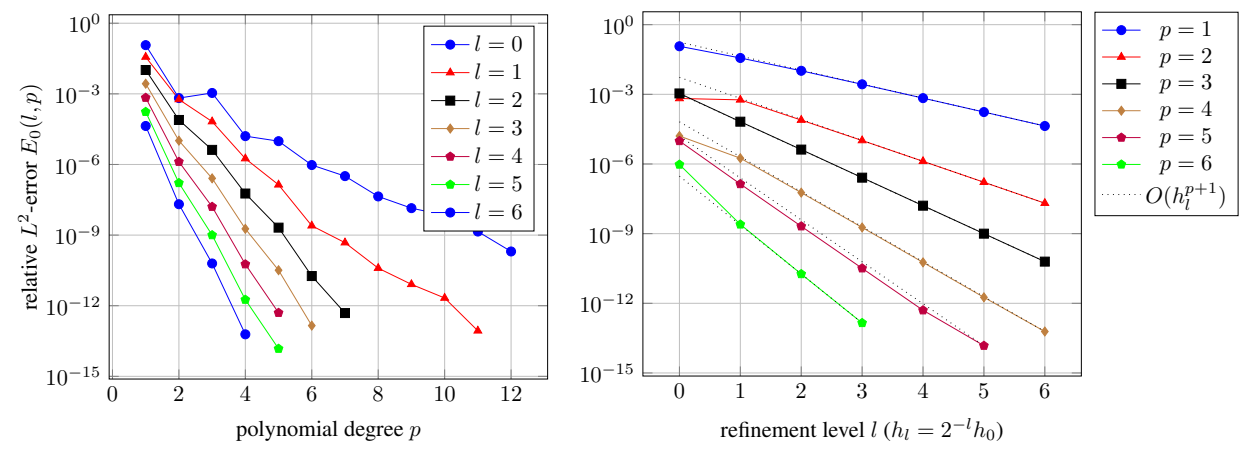

FIG. 4. Elliptic equilibrium: Relative $L^{2}$-error $E_{0}(l, p):=\left\|\psi_{h_{l}, p}-\psi_{\mathrm{E}}\right\|_{L^{2}(\Omega)} /\left\|\psi_{\mathrm{E}}\right\|_{L^{2}(\Omega)}$ for increasing polynomial refinement $p$ (left) and increasing mesh refinement level l (right).

and $\left(r_{\mathrm{E}}(s, t), z_{\mathrm{E}}(s, t)\right)$ is a parametrization of its level sets, i.e. $\psi_{\mathrm{E}}\left(r_{\mathrm{E}}(s, t), z_{\mathrm{E}}(s, t)\right)=$ $s$. With this we can find analytic expressions for geometric coefficients that are useful for numerical experiments:

$$
c_{\frac{1}{r^{2}}, \bar{\psi}_{\mathrm{E}}}(y)=\frac{6 \pi}{\sqrt{4-2 y}}, \quad c_{\frac{\left|\nabla \psi_{\mathrm{E}}\right|^{2}}{r^{2}}, \bar{\psi}_{\mathrm{E}}}(y)=\frac{24 \pi}{\sqrt{4-2 y}}-12 \pi .
$$

Convergence of $\psi_{\mathrm{E}}$. To begin with, we are using the Galerkin method to determine an approximation $\psi_{h, p}$ of $\psi_{\mathrm{E}}$ in $V_{h, p}$. The convergence estimates (28) are clearly reproduced by our experiments (see Figure 4).

Convergence of the geometric coefficients. In the following experiments we fix $P=$ 15 and study the convergence of the geometric coefficients. The convergence in both the $L^{2}$-norm and $H^{1}$-norm is in general better than predicted by Theorem 8 and Corollary 10. We observe a convergence like $h^{2}|\log (h)|^{2 / 3}$ for $p=1$ and like $h^{2 p}$ for $p>1$ for both coefficients $c_{\frac{1}{r^{2}}, \bar{\psi}_{\mathrm{E}}}$ and $\frac{c_{\left|\nabla \psi_{\mathrm{E}}\right|^{2}}, \bar{\psi}_{\mathrm{E}}}{r^{2}}$ (see Figure 5). Surprisingly, the additional consistency error by taking $f_{h, p}$ instead of $f$ in $\frac{c_{\left|\nabla \psi_{\mathrm{E}}\right|^{2}}}{r^{2}}, \bar{\psi}_{\mathrm{E}}$ does not seem to spoil the rate of convergence.

The experimental results highlight even more that the new method for computing the geometric coefficients is most powerful, when combined with an high order polynomial approximation of $\psi$. In this case the approximation error in $\psi$ decays very quickly and one achieves low error levels in the geometric coefficients despite the positive powers of $P$ in front of this contribution, see Theorem 8 and Corollary 10. Finally, our experiments suggest also that the high powers of the discretization parameter $P$ in the estimates of Theorem 8 and Corollary 10 are too pessimistic (see Figure 6). We observe in these examples a convergence like $P^{\frac{1}{2}}$ rather than $P^{4}$ for the error in $L^{2}(0,1)$. For the error in $H^{1}(0,1)$ we see a convergence like $P^{\frac{5}{2}}$ rather than $P^{6}$. Additionally, we see in the experiments the saturation levels for small $P$, which highlights that the discretization parameter $P$ needs to be balanced accordingly with $h$ and $p$, to achieve a desired accuracy level with minimal effort.

5.2.2. Application: Solov'ev Equilibrium. Finally, we study a more realistic example, taken from [21], that is a very popular and important benchmark case in computational plasma physics. It corresponds to the data $\psi^{\mathrm{bd}}=0, j(r, z)=\frac{1+\kappa^{2}}{\mu_{0} \kappa r_{0}^{3} q_{0}} r$ and

$$
\partial \Omega=\left\{(r, z): r^{2}=r_{0}^{2}+2 a r_{0} \cos (t), z=\kappa a \frac{r_{0}}{r} \sin (t), 0 \leq t \leq 2 \pi\right\},
$$

where the parameters $r_{0}$ and $q_{0}$ are the so called major radius and the safety factor at the magnetic axis, and the parameters $a$ and $\kappa$ the so called effective minor radius of the domain 

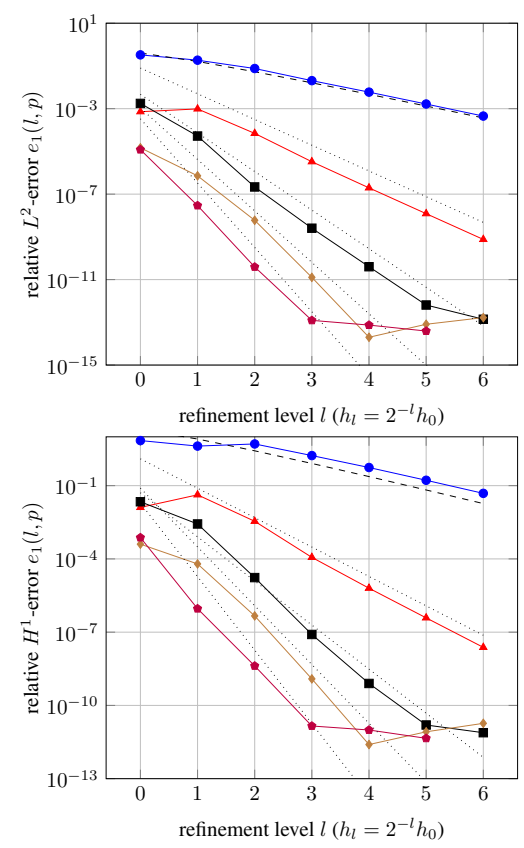
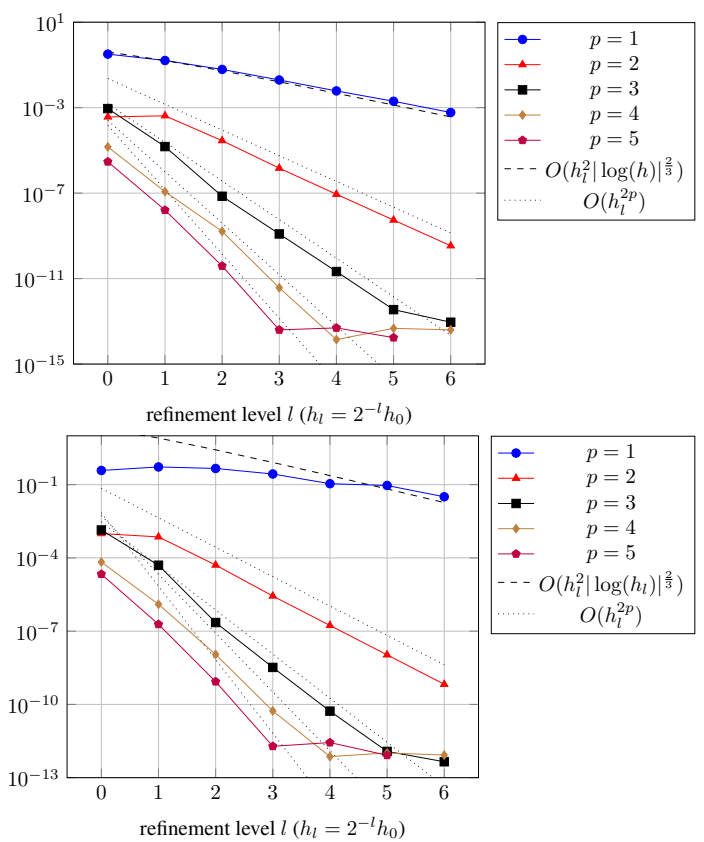

FIG. 5. Elliptic equilibrium: Relative $L^{2}$-error $e_{0}(l, p):=\left\|\widetilde{c}_{f, \bar{\psi}_{h_{l}, p}}-c_{f, \bar{\psi}_{\mathrm{E}}}\right\|_{L^{2}(0,1)} /\left\|c_{f, \bar{\psi}_{\mathrm{E}}}\right\|_{L^{2}(0,1)}$ (top) and $H^{1}$-error $e_{1}(l, p):=\left\|\widetilde{c}_{f, \bar{\psi}_{h_{l}, p}^{P}}-c_{f, \bar{\psi}_{\mathrm{E}}}\right\|_{H^{1}(0,1)} /\left\|c_{f, \bar{\psi}_{\mathrm{E}}}\right\|_{H^{1}(0,1)}$ (bottom) for $f=1 / r^{2}$ (left) and $f_{h_{l}, p}=\left|\nabla \psi_{h_{l}, p}\right|^{2} / r^{2}$ (right) as function of the mesh refinement level l for $P=15$ fixed.

and elongation of the domain. Then the solution of (26) is

$$
\psi_{\mathrm{S}}(r, z)=-\frac{\kappa}{2 r_{0}^{3} q_{0}}\left(\frac{1}{4}\left(r^{2}-r_{0}^{2}\right)^{2}+\frac{1}{\kappa^{2}} r^{2} z^{2}-a^{2} r_{0}^{2}\right) .
$$

In our experiments we have chosen the parameter values $r_{0}=1, a=0.32, \kappa=1.7$ and $q_{0}=1.0$.

Convergence of the geometric coefficients. For this example we do not have analytic values of the various geometric coefficients $\widetilde{c}_{f, \bar{\psi}_{\mathrm{S}}}$ with $f=1 / r^{2}$ and $f=|\nabla \psi|^{2} / r^{2}$. Hence, we explore the convergence behavior using a reference solution $\widetilde{c}_{f, \bar{\psi}_{\mathrm{S}}}^{\mathrm{ref}}:=\widetilde{c}_{f, \bar{\psi}_{h_{2}, 16}}^{15}$ computed at a discretization with $P=15$ for the discretization of the geometric coefficient and $p=16$ on a two times refined mesh for the discretization of $\psi_{S}$.

In Figure 7 we plot the error for refinement in the polynomial degree $p$ and in the mesh refinement level $l$. The polynomial degree is held fixed at $P=15$. Here again we observe spectral convergence for geometric coefficients $c_{f, \bar{\psi}_{S}}$ that involve functions $f$ independent of $\psi_{\mathrm{S}}$ or functions that are dependent of $\psi_{\mathrm{S}}$. To achieve a certain error level with a minimal number of degrees of freedom for the approximation $\psi_{h, p}$ it is advantageous to chose larger values for $p$ rather than to work with very fine meshes. Figure 8 illustrates $\psi_{h_{l}, 1}$ on the initial mesh and after 1 and 2 refinements.

6. Conclusions. We presented a novel high-order accurate method for numerical integration of functions over hypersurfaces. This method avoids the use of explicit parametrizations of the hypersurface, that are in general not available. Both the numerical analysis and 

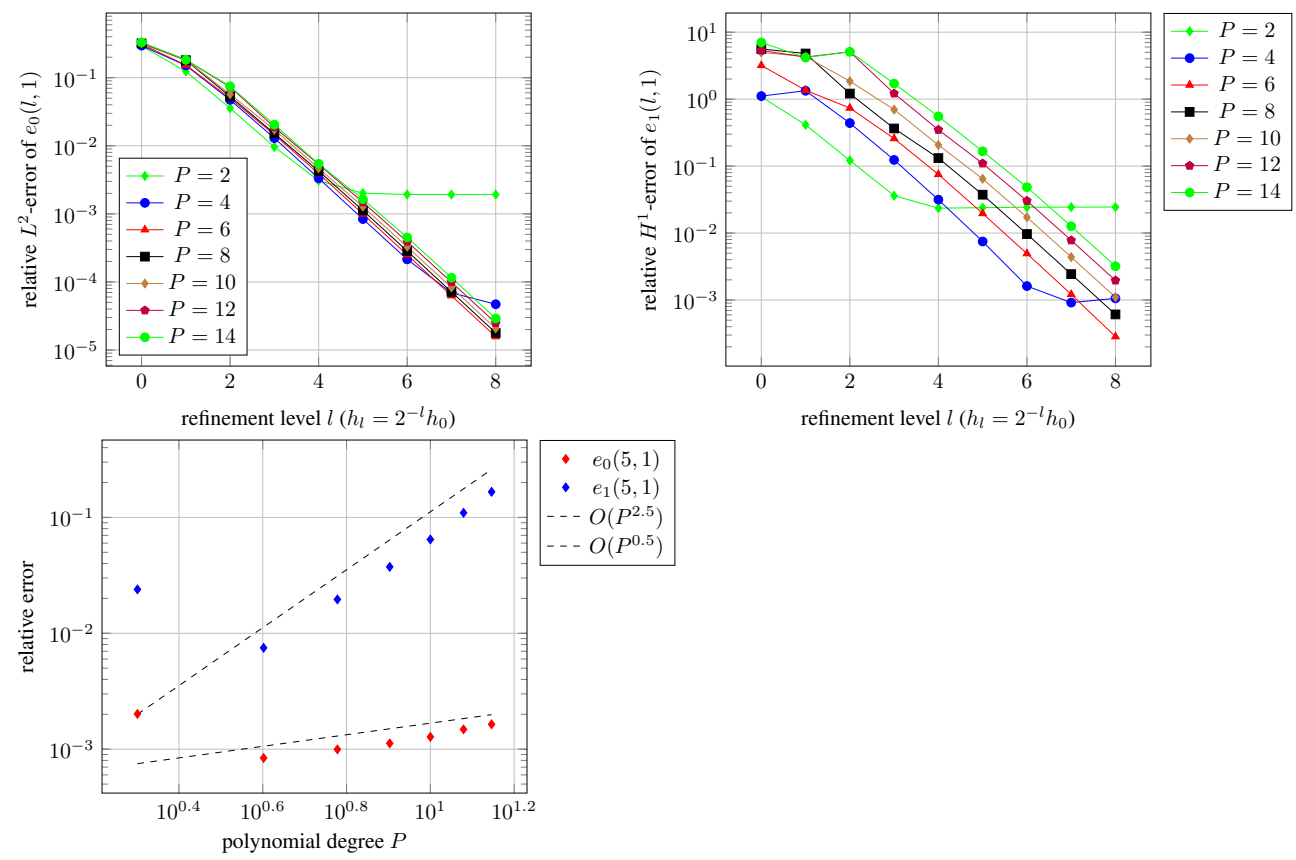

FIG. 6. Elliptic equilibrium: Relative $L^{2}$-error $e_{0}(l, 1):=\left\|\widetilde{c}_{f, \bar{\psi}_{h_{l}, 1}^{P}}-c_{f, \bar{\psi}_{\mathrm{E}}}\right\|_{L^{2}(0,1)} /\left\|c_{f, \bar{\psi}_{\mathrm{E}}}\right\|_{L^{2}(0,1)}$ (top left) and $H^{1}$-error $e_{1}(l, 1):=\left\|\widetilde{c}_{f, \bar{\psi}_{h_{l}, 1}^{P}}-c_{f, \bar{\psi}_{\mathrm{E}}}\right\|_{H^{1}(0,1)} /\left\|c_{f, \bar{\psi}_{\mathrm{E}}}\right\|_{H^{1}(0,1)}$ (top right) for $f=1 / r^{2}$ as a function of the refinement level $l$ of the mesh for different polynomial degrees $P$ and relative $L^{2}$-error $e_{0}(l, 1)$ and $H^{1}$-error $e_{1}(l, 1)$ as a function of the polynomial degree $P$ at fixed level $l=5$ (bottom).
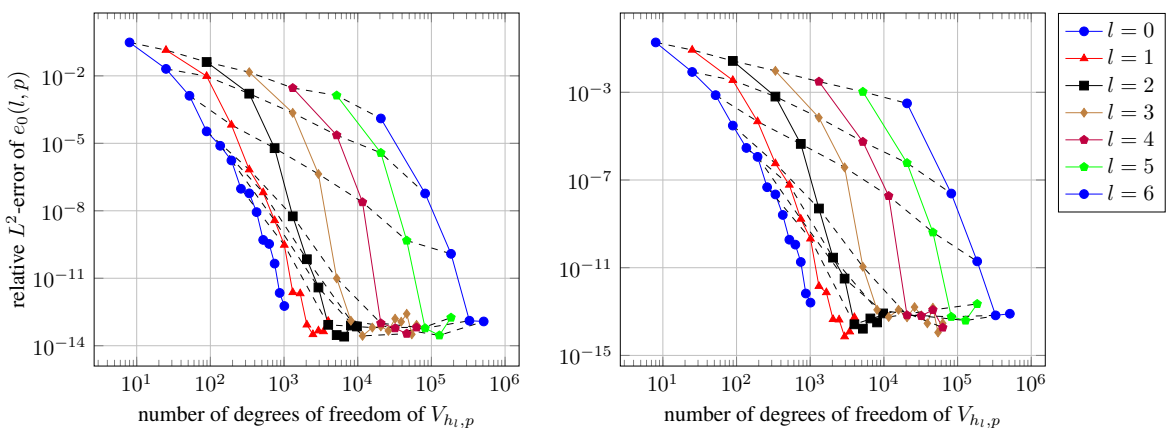

FIG. 7. Solov'ev equilibrium: Relative $L^{2}$-error $e_{0}(l, p):=\left\|\widetilde{c}_{f, \bar{\psi}_{h_{l}, p}^{P}}^{P}-c_{f, \psi_{\mathrm{S}}}^{\mathrm{ref}}\right\|_{L^{2}(0,1)} /\left\|c_{f, \bar{\psi}_{\mathrm{S}}}^{\mathrm{ref}}\right\|_{L^{2}(0,1)}$ for $f=1 / r^{2}$ (left) and $f_{h_{l}, p}=\left|\nabla \psi_{h_{l}, p}\right|^{2} / r^{2}$ (right) for refinement in polynomial degree $p$ and mesh refinement level l for $P=15$ fixed.

our numerical examples provide evidence of fast convergence. Moreover, the method is easy to implement in a pre-existing finite element solver as it requires no extra geometric data structures or non-linear solvers needed to explicitly resolve level sets. The only non-standard component is the evaluation of test functions concatenated with the (possibly discrete) level set function in the right hand side of the weak formulation. While the motivation of this work clearly stems from plasma equilibrium modeling in nuclear fusion science, we want to highlight that integrals over hypersurfaces are a standard task in many engineering problems and 

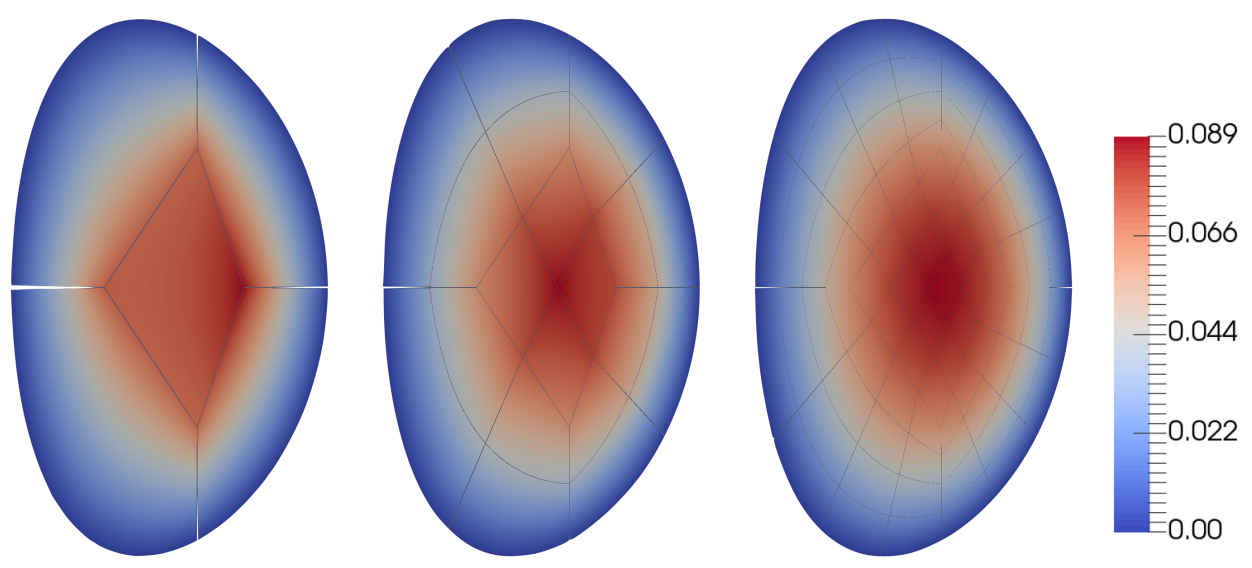

FIG. 8. Solov'ev equilibrium problem: Numerical solution $\psi_{h_{l}, 1}$ on the initial mesh $(l=0$, left $)$ and after $l=1$ (middle), $l=2$ (right) geometric refinements via bisection along all coordinate directions $\left(h_{l}=2^{-l} h_{0}\right)$. The boundary $\partial \Omega$ is resolved exactly in all meshes by virtue of splitting the explicit parametrization in (30) at element boundaries and applying transfinite interpolation.

an important building block in numerical methods, such as the boundary element method, fictitious domains method, mortar element methods or level set methods. Shape optimization problems or fluid structure interaction obviously require the approximation of hypersurface integrals. As such the proposed method can have implications in many different areas.

7. Acknowledgments. The authors thank the referees for their comments and suggestions. These led to a significantly improved revised version. We thank Rolf Krause (Università della Svizzera Italiana, Lugano, Switzerland) for pointing us to improved $L^{\infty}(0,1)$ estimates. We also thank Jacques Blum (Université Côte d'Azur, Nice, France) for the financial support of the first author during his stay at the Laboratoire J.A. Dieudonné.

Appendix A. A few regularity results for hypersurface integrals. The assumption $g_{f, \bar{\psi}} \in H^{k}(0,1)$ in Theorem 4 is fairly strong. First of all it is obvious that $g_{f, \bar{\psi}}$ might lose regularity in the neighborhood of values $y \in[0,1]$ where $\psi$ has a stationary point, i.e. $\nabla \bar{\psi}(\mathbf{x})=0$ and $\bar{\psi}(\mathbf{x})=y$. Second, it can be expected that a loss of regularity $\psi$ influences also the regularity of the hypersurface integrals. To our knowledge it is an open problem to quantify precisely how the regularity of $\psi$ influences the regularity of $g_{f, \bar{\psi}}$. There is also very few theory available explaining how stationary points of $\psi$ influence the regularity of $g_{f, \bar{\psi}}$. The only result, we are aware of, is due to G. Vigfússon, stated in [33, Theorem 2].

THEOREM 14 (G. Vigfússon 1979). Assume that the boundary $\partial \Omega \in C^{\infty}$ is arbitrarily smooth and that $\bar{\psi}$ has only one stationary point in $\bar{\Omega}$, that is the maximum of $\bar{\psi}$ in $\Omega$ and $\bar{\psi}_{\mid \partial \Omega} \equiv \psi^{1} \in \mathbb{R}$, then

$$
g_{f|\nabla \bar{\psi}|^{-1}, \bar{\psi}} \in C^{n}([0,1]), \quad \text { if } \quad f \in C^{2 n}(\Omega) \text { and } \bar{\psi} \in C^{2 n+2}(\Omega) .
$$

Proof. The proof for $n=0$ and $n=1$ follows from various results in [32] and is based on a technique called elliptic expansions that compares the contour lines of $\bar{\psi}$ with ellipses and quantifies the deviation. The Theorems 9 and 10 in [32, p. 62] provide

$$
G_{|\nabla \bar{\psi}|^{-1}, \bar{\psi}}(\cdot):=\int_{0} g_{|\nabla \bar{\psi}|^{-1}, \bar{\psi}}\left(y^{\prime}\right) d y^{\prime} \in C^{n+1}([0,1]) \quad \text { if } \quad \bar{\psi} \in C^{2 n+2}(\Omega),
$$


with $n=0, n=1$ or $n=2$. Furthermore, another theorem in [32, p. 75] states

$$
\frac{g_{f|\nabla \bar{\psi}|^{-1}, \bar{\psi}}}{g_{|\nabla \bar{\psi}|^{-1}, \bar{\psi}}} \in C^{n}([0,1]), \quad \text { if } \quad f \in C^{2 n}(\Omega) \text { and } \bar{\psi} \in C^{2 n+1}(\Omega),
$$

with $n=0$ or $n=1$ and the assertion of Theorem 14 follows.

Such a smoothness result is not true if $\bar{\psi}$ has saddle points in $\Omega$ as we see for the example in the following remark.

REMARK 15. We consider $\Omega=[0,1]^{2}, \bar{\psi}(r, z)=1-r z$ and $f(r, z)=\sum_{i, j \geq 0} a_{i j} r^{i} z^{j}$ with $a_{i j} \in \mathbb{R} . \bar{\psi}(r, z)$ has a saddle point at $\left(r_{X}, z_{X}\right)=(0,0)$ with $y_{X}=1$. We compute for $f_{1}(r, z)=r^{n} z^{m}, n, m \in \mathbb{N}, n \neq m:$

$$
\begin{aligned}
G_{f_{1}|\nabla \bar{\psi}|-1, \bar{\psi}}(y) & =\int_{1-y}^{1}\left(\int_{(1-y) / z}^{1} r^{n} z^{m} d r\right) d z \\
& =\frac{(m-n)-(m+1)(1-y)^{n+1}+(n+1)(1-y)^{m+1}}{(m+1)(n+1)(m-n)}
\end{aligned}
$$

and for $f_{2}(r, z)=r^{n} z^{n}, n \in \mathbb{N}$ :

$$
\begin{aligned}
G_{f_{2}|\nabla \bar{\psi}|^{-1}, \bar{\psi}}(y) & =\int_{1-y}^{1}\left(\int_{(1-y) / z}^{1} r^{n} z^{n} d r\right) d z \\
& =\frac{1-(1-y)^{n+1}}{(n+1)^{2}}+\frac{(1-y)^{n+1} \log (1-y)}{n+1},
\end{aligned}
$$

which yields

$$
G_{f_{1}|\nabla \bar{\psi}|^{-1}, \bar{\psi}}^{\prime}(y)=g_{f_{1}|\nabla \bar{\psi}|^{-1}, \bar{\psi}}(y)=\frac{(1-y)^{n}-(1-y)^{m}}{m-n} .
$$

and

$$
G_{f_{2}|\nabla \bar{\psi}|^{-1}, \bar{\psi}}^{\prime}(y)=g_{f_{2}|\nabla \bar{\psi}|^{-1}, \bar{\psi}}(y)=-\log (1-y)(1-y)^{n} .
$$

Hence we find

$$
g_{f|\nabla \bar{\psi}|^{-1}, \bar{\psi}}(y)=\sum_{i, j \geq 0 i \neq j} a_{i j} \frac{(1-y)^{i}-(1-y)^{j}}{j-i}-\log (1-y) \sum_{i \geq 0} a_{i i}(1-y)^{i} .
$$

In the neighborhood of the saddle point $y=y_{X}(=1)$ the hypersurface integral $g_{f|\nabla \bar{\psi}|^{-1}, \bar{\psi}}(y)$ behave like $\log \left(y_{X}-y\right)$ whenever $a_{00} \neq 0$. In particular, $g_{f|\nabla \bar{\psi}|^{-1}, \bar{\psi}}\left(y_{X}\right)$ is unbounded, while the quotient $g_{f|\nabla \bar{\psi}|^{-1}, \bar{\psi}}\left(y_{X}\right) / c_{1, \bar{\psi}}\left(y_{X}\right)$ is bounded:

$$
\lim _{y \rightarrow y_{X}} \frac{g_{f|\nabla \bar{\psi}|^{-1}, \bar{\psi}}(y)}{g_{|\nabla \bar{\psi}|^{-1}, \bar{\psi}}(y)}=f\left(r_{X}, z_{X}\right)=a_{00} .
$$

Appendix B. Auxiliary results for the numerical analysis. In this section we collect some auxiliary results. 
Lemma 16. Let $\widehat{\omega}_{j} \in \mathbb{R}$ and $\widehat{x}_{j} \in \mathbb{R}^{n}, 1 \leq j \leq R$ be weights and quadrature points of a quadrature rule over an reference element $\widehat{\widehat{K}} \subset \mathbb{R}^{n}$ with diameter $\operatorname{diam}(\widehat{K})=1$ that is exact for polynomials of degree q, i.e.

$$
\widehat{E}(\widehat{p}):=\int_{\widehat{K}} \widehat{p}(\widehat{\mathbf{x}}) d \widehat{\mathbf{x}}-\sum_{j=1}^{R} \widehat{\omega}_{j} \widehat{p}\left(\widehat{\mathbf{x}}_{j}\right)=0 \quad \forall \widehat{p} \in \mathcal{P}_{q}(\widehat{K}),
$$

where $\mathcal{P}_{q}(\widehat{K})$ is the set of polynomials of degree $q$ over $\widehat{K}$. Let $K \subset \mathbb{R}^{n}$ be the image of $\widehat{K}$ under an affine transformation. Then for all $\ell$ with $q+1>\ell$

$$
\begin{aligned}
& \mid \int_{K} g(\mathbf{x}) \xi(\mathbf{x}) d \mathbf{x}-|K| \sum_{j=1}^{R} \omega_{j} g\left(\mathbf{x}_{j}\right) \xi\left(\mathbf{x}_{j}\right) \mid \\
& \leq C \operatorname{diam}(K)^{q-l+1}|K|^{\frac{1}{2}}\|g\|_{W^{q-\ell+1, \infty}(K)}\|\xi\|_{L^{2}(K)}, \forall \xi \in \mathcal{P}_{\ell}(K) .
\end{aligned}
$$

Proof. We introduce

$$
E(p):=\int_{K} p(\mathbf{x}) d \mathbf{x}-|K| \sum_{j=1}^{R} \widehat{\omega}_{j} p\left(\mathbf{x}_{j}\right)
$$

and have $(\widehat{p}=p \circ \Phi)$

$$
\begin{aligned}
E(g \xi)=|K| \widehat{E}(\widehat{g} \widehat{\xi}) & \leq C|K|\|\widehat{g} \widehat{\xi}\|_{L^{\infty}(\widehat{K})} \\
& \leq C|K|\|\widehat{g}\|_{L^{\infty}(\widehat{K})}\|\widehat{\xi}\|_{L^{\infty}(\widehat{K})} \\
& \leq C|K|\|\widehat{g}\|_{W^{q-\ell+1, \infty}(\widehat{K})}\|\widehat{\xi}\|_{L^{\infty}(\widehat{K})} \\
& \leq C\left|K\left\|\left.\widehat{g}\right|_{W^{q-\ell+1, \infty}(\widehat{K})}\right\| \widehat{\xi} \|_{L^{\infty}(\widehat{K})}\right. \\
& \leq C\left|K\left\|\left.\widehat{g}\right|_{W^{q-\ell+1, \infty}(\widehat{K})}\right\| \widehat{\xi} \|_{L^{2}(\widehat{K})}\right. \\
& \leq C \operatorname{diam}(\mathrm{K})^{q-\ell+1}|K|^{\frac{1}{2}}|g|_{W^{q-\ell+1, \infty}(K)}\|\xi\|_{L^{2}(K)}
\end{aligned}
$$

where in the third step we use $W^{q-l+1, \infty}(\widehat{K}) \hookrightarrow C^{0}(\widehat{K})$ since $q-l+1>0$, the fourth step is Bramble-Hilbert, the fifth step equivalence of $\|\cdot\|_{L^{\infty}(\widehat{K})}$ and $\|\cdot\|_{L^{2}(\widehat{K})}$ on $\mathcal{P}(\widehat{K})$ and the sixth step follows from scaling due to back transformation.

LEMma 17 ( $L^{\infty}(0,1)$-bounds of polynomials). For any $\lambda \in \mathcal{P}_{P}(0,1)$ it holds

$$
\|\lambda\|_{L^{\infty}(0,1)} \leq(P+1)\|\lambda\|_{L^{2}(0,1)} .
$$

Proof. Taking the (shifted) Legendre polynomials $L_{k}, k=0, \ldots, P$ as $L^{2}(0,1)$-orthogonal basis of $\mathcal{P}_{P}(0,1)$ with $\left\|L_{k}\right\|_{L^{2}(0,1)}=1 / \sqrt{2 k+1}$ and the fact that all $L_{k}$ are all in $[0,1]$ bounded by 1 [1, Chap. 22] and so $\left\|L_{k}\right\|_{L^{\infty}(0,1)} \leq 1=\sqrt{2 k+1}\left\|L_{k}\right\|_{L^{2}(0,1)}$ we find for $\lambda=\sum_{k=0}^{P} \lambda_{k} L_{k}$ that

$$
\|\lambda\|_{L^{\infty}(0,1)} \leq \sum_{k=0}^{P}\left|\lambda_{k}\right|\left\|L_{k}\right\|_{L^{\infty}(0,1)} \leq \sum_{k=0}^{P} \sqrt{2 k+1}\left|\lambda_{k}\right|\left\|L_{k}\right\|_{L^{2}(0,1)} \leq(P+1)\|\lambda\|_{L^{2}(0,1)},
$$

where we used the orthogonality of $L_{k}$ in the last step, and the proof is complete. 
LeMma 18. For any functions $\psi_{ \pm} \in L^{\infty}(\Omega)$ it holds

$$
\begin{aligned}
& \left|\inf _{\mathbf{x} \in \Omega} \psi_{+}(\mathbf{x})-\inf _{\mathbf{x} \in \Omega} \psi_{-}(\mathbf{x})\right| \leq 2\left\|\psi_{+}-\psi_{-}\right\|_{L^{\infty}(\Omega)} \\
& \left|\sup _{\mathbf{x} \in \Omega} \psi_{+}(\mathbf{x})-\sup _{\mathbf{x} \in \Omega} \psi_{-}(\mathbf{x})\right| \leq 2\left\|\psi_{+}-\psi_{-}\right\|_{L^{\infty}(\Omega)} .
\end{aligned}
$$

Proof. We prove the first assertion and remark that the second follows analogously. Without loss of generality let $\epsilon:=\inf _{\mathbf{x} \in \Omega} \psi_{+}(\mathbf{x})-\inf _{\mathbf{x} \in \Omega} \psi_{-}(\mathbf{x}) \geq 0$. Then, there exists $\mathbf{x}^{\star} \in \Omega$ such that

$$
\psi_{-}\left(\mathbf{x}^{\star}\right)+\frac{\epsilon}{2} \leq \inf _{\mathbf{x} \in \Omega} \psi_{+}(\mathbf{x}) \leq \psi_{+}\left(\mathbf{x}^{\star}\right)
$$

Hence,

$$
\epsilon \leq 2\left(\psi_{+}\left(\mathbf{x}^{\star}\right)-\psi_{-}\left(\mathbf{x}^{\star}\right)\right) \leq 2\left\|\psi_{+}-\psi_{-}\right\|_{L^{\infty}(\Omega)},
$$

and the proof is complete.

Lemma 19. Let $|\cdot|_{\Omega}$ be a seminorm on $\Omega, f_{ \pm}, \psi_{ \pm} \in L^{\infty}(\Omega)$ such that $\left|\psi_{ \pm}\right|_{\Omega}<\infty$, $\psi^{0}\left(\psi_{ \pm}\right)=\sup _{\mathbf{x} \in \Omega} \psi_{ \pm}, \psi^{1}\left(\psi_{ \pm}\right)=\inf _{\mathbf{x} \in \Omega} \psi_{ \pm}, \psi^{0}\left(\psi_{ \pm}\right)>\psi^{1}\left(\psi_{ \pm}\right)$and $\bar{\psi}_{ \pm}=\left(\psi_{ \pm}-\right.$ $\left.\psi^{0}\left(\psi_{ \pm}\right)\right) /\left(\psi^{1}\left(\psi_{ \pm}\right)-\psi^{0}\left(\psi_{ \pm}\right)\right)$. Then, there exists a constant $C$ independent of $\psi_{-}$such that

$$
\left|\bar{\psi}_{+}-\bar{\psi}_{-}\right|_{\Omega} \leq C \frac{\left|\psi_{+}-\psi_{-}\right|_{\Omega}+\| \psi_{+}-\left.\psi_{-}\right|_{L^{\infty}(\Omega)}}{\psi^{0}\left(\psi_{-}\right)-\psi^{1}\left(\psi_{-}\right)}
$$

If moreover $\psi_{ \pm} \in H^{1}(\Omega)$ then with a constant $C$ independent of $\psi_{-}$it holds

$$
\left.\left|f_{+}\right| \nabla \bar{\psi}_{+}\left|-f_{-}\right| \nabla \bar{\psi}_{-}\right|_{\Omega} \leq C \frac{\left.\left|f_{+}\right| \nabla \psi_{+}\left|-f_{-}\right| \nabla \psi_{-}\right|_{\Omega}+\left\|\psi_{+}-\psi_{-}\right\|_{L^{\infty}(\Omega)}}{\psi^{0}\left(\psi_{-}\right)-\psi^{1}\left(\psi_{-}\right)} .
$$

Proof. The first estimate follows by the equality

$$
\begin{array}{r}
\left(\psi^{1}\left(\psi_{-}\right)-\psi^{0}\left(\psi_{-}\right)\right)\left(\bar{\psi}_{+}-\bar{\psi}_{-}\right)=\left(\psi_{+}-\psi_{-}\right)-\frac{\psi_{+}-\psi^{1}\left(\psi_{+}\right)}{\psi^{0}\left(\psi_{+}\right)-\psi^{1}\left(\psi_{+}\right)}\left(\psi^{0}\left(\psi_{+}\right)-\psi^{0}\left(\psi_{-}\right)\right) \\
-\frac{\psi_{+}-\psi^{0}\left(\psi_{+}\right)}{\psi^{1}\left(\psi_{+}\right)-\psi^{0}\left(\psi_{+}\right)}\left(\psi^{1}\left(\psi_{+}\right)-\psi^{1}\left(\psi_{-}\right)\right),
\end{array}
$$

the triangle inequality and Lemma 18. Similarly, with the equality

$$
\begin{aligned}
\left(\psi^{1}\left(\psi_{-}\right)-\psi^{0}\left(\psi_{-}\right)\right) & \left(f_{+}\left|\nabla \bar{\psi}_{+}\right|-f_{-}\left|\nabla \bar{\psi}_{-}\right|\right)=f_{+}\left|\nabla \psi_{+}\right|-f_{-}\left|\nabla \psi_{-}\right| \\
& -\frac{f_{+}\left|\nabla \psi_{+}\right|}{\psi^{1}\left(\psi_{+}\right)-\psi^{0}\left(\psi_{+}\right)}\left(\psi^{1}\left(\psi_{+}\right)-\psi^{1}\left(\psi_{-}\right)+\psi^{0}\left(\psi_{+}\right)-\psi^{0}\left(\psi_{-}\right)\right)
\end{aligned}
$$

we obtain the second estimate.

\section{REFERENCES}

[1] M. Abramowitz AND I. A. STEgun, Handbook of mathematical functions with formulas, graphs, and mathematical tables, National Bureau of Standards Applied Mathematics Series, 1964.

[2] R. A. AdAms, Sobolev spaces, Academic Press, 1975.

[3] K. AtKinson And E. Venturino, Numerical evaluation of line integrals, SIAM J. Numer. Anal., 30 (1993), pp. 882-888, doi:10.1137/0730046. 
[4] P. G. Ciarlet, The Finite Element Method for Elliptic Problems, North-Holland Publishing Co., 1978.

[5] P. G. Ciarlet, Basic error estimates for elliptic problems, Finite Element Methods (Part 1), Handbook of Numerical Analysis, North-Holland Publishing Co., 1991.

[6] L. Demkowicz, Computing with hp-adaptive finite elements. Vol. 1, Chapman \& Hall/CRC Applied Mathematics and Nonlinear Science Series, Chapman \& Hall/CRC, Boca Raton, FL, 2007, doi:10.1201/9781420011692. One and two dimensional elliptic and Maxwell problems, With 1 CDROM (UNIX).

[7] B. ENGQUIST, A.-K. TORNBERG, AND R. TSAI, Discretization of Dirac delta functions in level set methods, J. Comput. Phys., 207 (2005), pp. 28-51, doi:10.1016/j.jcp.2004.09.018.

[8] H. FedERER, Geometric measure theory, Die Grundlehren der mathematischen Wissenschaften, Band 153, Springer-Verlag New York Inc., New York, 1969.

[9] P. Frauenfelder And C. LAGE, Concepts - An Object-Oriented Software Package for Partial Differential Equations, ESAIM: Math. Model. Numer. Anal., 36 (2002), pp. 937-951, doi:10.1051/m2an:2002036.

[10] T.-P. FRIES AND S. OMEROVIC, Higher-order accurate integration of implicit geometries, Internat. J. Numer. Methods Engrg., 106 (2016), pp. 323-371, doi:10.1002/nme.5121. nme.5121.

[11] J. P. Goedbloed AND S. PoedTS, Principles of magnetohydrodynamics: with applications to laboratory and astrophysical plasmas, Cambridge University Press, 2004.

[12] H. Grad And J. Hogan, Classical diffusion in a tokamak, Phys. Rev. Lett., 24 (1970), pp. 1337-1340, doi:10.1103/PhysRevLett.24.1337.

[13] H. GRAD AND H. RUBIN, Hydromagnetic equilibria and force-free fields, Proceedings of the 2nd UN Conf. on the Peaceful Uses of Atomic Energy, 31 (1958), p. 190, http://www-naweb.iaea.org/napc/physics/ 2ndgenconf/data/Proceedings1958/papersVol31/Paper25_Vol31.pdf.

[14] J. Grande, Analysis of Highly Accurate Finite Element Based Algorithms for Computing Distances to Level Sets, SIAM J. Numer. Anal., 55 (2017), pp. 76-399

[15] S. C. JARdin, Computational methods in plasma physics, Boca Raton, FL : CRC Press/Taylor \& Francis, 2010.

[16] P. Knabner And L. Angermann, Numerical methods for elliptic and parabolic partial differential equations, vol. 44 of Texts in Applied Mathematics, Springer-Verlag, New York, 2003.

[17] C. LEHRENFELD, High order unfitted finite element methods on level set domains using isoparametric mappings, Comput. Methods Appl. Mech. Eng., 300(2016), pp. 716-733.

[18] J.-L. Lions And J. Peetre, Sur Une Classe D'Espaces D'Interpolation, Inst. Hautes Études Sci. Publ. Math., 1 (1968), pp. 1618-1913.

[19] W. E. LORENSEN AND H. E. CLINE, Marching cubes: A high resolution 3d surface construction algorithm, SIGGRAPH Comput. Graph., 21 (1987), pp. 163-169, doi:10.1145/37402.37422.

[20] R. LÜST AND A. SCHLÜTER, Axialsymmetrische magnetohydrodynamische Gleichgewichtskonfigurationen., Z. Naturforsch. A, 12 (1957), pp. 850-854.

[21] H. LÜTJEns, A. Bondeson, AND O. SAUter, The CHEASE code for toroidal MHD equilibria, Comput. Phys. Commun., 97 (1996), pp. 219 - 260, doi:http://dx.doi.org/10.1016/0010-4655(96)00046-X.

[22] C. Min AND F. GiBOU, Geometric integration over irregular domains with application to level-set methods, J. Comput. Phys., 226 (2007), pp. 1432 - 1443, doi:http://dx.doi.org/10.1016/j.jcp.2007.05.032.

[23] T. S. Newman AND H. YI, A survey of the marching cubes algorithm, Computers \& Graphics, 30 (2006), pp. 854 - 879, doi:http://dx.doi.org/10.1016/j.cag.2006.07.021.

[24] B. A. PAYne AND A. W. Toga, Surface mapping brain function on 3D models, IEEE Computer Graphics and Applications, 10 (1990), pp. 33-41, doi:10.1109/38.59034.

[25] N. RAY, D. WANG, X. JIAO, AND J. GLIMM, High-order numerical integration over discrete surfaces, SIAM J. Numer. Anal., 50 (2012), pp. 3061-3083, doi:10.1137/110857404.

[26] R.I. SAYE, High-Order Quadrature Methods for Implicitly Defined Surfaces and Volumes in Hyperrectangles, SIAM J. Sci. Comp., 37 (2015), pp. A993-A1019.

[27] K. SCHMIDT AND P. KAUF, Computation of the band structure of two-dimensional photonic crystals with hp finite elements, Comput. Methods Appl. Mech. Engrg., 198 (2009), pp. 1249-1259.

[28] C. SCHWAB, $p$ - and hp- finite element methods : theory and applications in solid and fluid mechanics, Clarendon Press, Oxford, 2004.

[29] V. D. ShAFRANOv, On magnetohydrodynamical equilibrium configurations, Soviet Journal of Experimental and Theoretical Physics, 6 (1958), p. 545, http://adsabs.harvard.edu/abs/1958JETP....6..545S.

[30] P. SHIRLEY AND A. TUCHMAN, A polygonal approximation to direct scalar volume rendering, SIGGRAPH Comput. Graph., 24 (1990), pp. 63-70, doi:10.1145/99308.99322.

[31] L. TARTAR, An introduction to Sobolev spaces and interpolation spaces, Springer Verlag, Berlin Heidelberg, 2007.

[32] G. VIGfússon, The averaged Green function with applications to quasi-static plasma equilibrium, $\mathrm{PhD}$ thesis, New York University, 1977.

[33] G. Vigfússon, The queer differential equations for adiabatic compression of plasma, Bull. Amer. Math. Soc. (N.S.), 1 (1979), pp. 778-781, doi:10.1090/S0273-0979-1979-14664-0. 
[34] M. WAng, C. Engström, K. Schmidt, And C. Hafner, On high-order FEM applied to canonical scattering problems in plasmonics, J. Comput. Theor. Nanosci., 8 (2011), pp. 1-9. 